\title{
Discrete-Time Nonlinear Controller Synthesis by Input/Output Linearization
}

\author{
Masoud Soroush and Costas Kravaris \\ Dept. of Chemical Engineering, The University of Michigan, Ann Arbor, MI 48109
}

\begin{abstract}
This work concerns the synthesis of discrete-time nonlinear controllers for nonlinear processes that make the closed-loop system linear in an input/output sense. The synthesis of state feedback controllers is studied first, followed by the synthesis of dynamic output feedback controllers. Both problems are solved within the globally linearizing control (GLC) framework. Precise theoretical connections between the derived controllers and model algorithmic control (MAC) are established. The theory is illustrated by a chemical reactor example.
\end{abstract}

\section{Introduction}

The area of nonlinear process control has received considerable attention during the past five years because of the realization that linear controllers are inadequate even for moderately nonlinear processes and the availability of new powerful tools. There have been several recent review articles aiming at giving a perspective on the present status of the area and trace further directions (McLellan et al., 1990; Kravaris and Kantor, 1990a,b; Bequette, 1991; Biegler and Rawlings, 1991; Kravaris and Arkun, 1991). So far, the two major research directions have been the model-predictive approach and the geometric approach. The key advantage of model-predictive control (MPC) has been its appealing intuitive interpretation, whereas the key advantage of the geometric approach has been its solid theoretical justification.

MPC algorithms have been used in industry since the late 70s [model algorithmic control (Richalet et al., 1978; Mehra and Rouhani, 1980; and Mehra et al., 1980) and dynamic matrix control (Cutler and Ramaker, 1979; Prett and Gillette, 1979)]. Garcia and Morari (1982) reviewed different versions of MPC, put them into perspective and connected them with theoretical $z$-domain methods via the internal model control (IMC) structure. At the same time, a continuous-time linear IMC structure was developed, which possessed all the theoretical properties of the discrete one, without any model-predictive algorithms being available in continuous time along the same lines. Later, the continuous-time version of the IMC was extended to nonlinear systems using an input/output operator formalism and numerical techniques for operator inversion (Economou et al., 1986; Li et al., 1990). In the past six years, there has been a growing research activity aiming at developing nonlinear MPC methods, primarily through direct nonlinear extension of the linear MPC techniques (Biegler and Rawlings, 1991; Henson and Seborg, 1991 b; Hernandez and Arkun, 1991; Hidalgo and Brosilow, 1990; Pathwardhan et al., 1990; Sistu and Bequette, 1991).

Geometric process control methods have evolved after about a decade of research on the mathematical characteristics of continuous-time nonlinear systems, using techniques from differential geometry. The system theoretic properties of continuous-time nonlinear systems are now well-understood (Isidori, 1989; Nijmeijer and van der Schaft, 1990), and they provide the theoretical foundations for nonlinear controller design. Based on the mathematical. tools and concepts from the differential geometric framework, the globally linearized control (GLC) method (Kravaris and Chung, 1987; Kravaris and Soroush, 1990) was developed as a nonlinear controller synthesis method in a continuous-time setting. The GLC was interpreted from the point of view of input/output operators and IMC (Daoutidis and Kravaris, 1992; Henson and Seborg, 1991a), and recently was implemented experimentally to control polymerization reactors (Soroush and Kravaris, 1992a,b).

In the late $80 \mathrm{~s}$, a major research effort was started extending the continuous-time geometric notions and results to discretetime nonlinear systems, and thus developing a discrete-time analog of geometric nonlinear systems theory. For instance, Grizzle (1986) studied the local input-output decoupling problem for discrete-time nonlinear systems, by employing invariant and locally-controlled invariant distributions. Monaco and Normand-Cyrot (1988) investigated the behavior of the zero dynamics of discrete-time nonlinear systems. Glad (1988) also studied the zero dynamics of discrete-time nonlinear systems and developed connections between the minimum-phase prop- 
erties of a discrete system and the internal stability of an output dead-beat controller. Monaco et al. (1989) addressed the problem of input-output decoupling in discrete time via static and dynamic feedback and gave necessary and sufficient conditions which ensure static decoupling and left invertibility of each input-output channel. A review of available results in discretetime nonlinear systems theory can be found in the recent monograph by Nijmeijer and van der Schaft (1990).

In the process control literature, a discrete-time GLC method was developed by Stubbs and Svoronos (1989) for discretetime input/output models expressed in terms of a single difference equation which is affine in the input. Similar results were presented very recently (Hernandez and Arkun, 1991) for polynomial ARMA models.

The scope of this article is twofold:

(1) Develop nonlinear geometric process control methods for general discrete-time state-space models within the conceptual and methodological framework of GLC (Kravaris and Chung, 1987; Daoutidis and Kravaris, 1992).

(2) Develop concrete theoretical connections between geometric and model-predictive approaches. In particular, we show that the discrete-time GLC-error feedback controller is a direct conceptual generalization of linear model algorithmic control (Richalet et al., 1978; Mehra et al., 1980; Mehra and Rouhani, 1980).

An earlier version of our discrete-time GLC results was presented at the AIChE meeting (Daoutidis et al., 1991).

In this article, some mathematical preliminaries are reviewed, including the definition of relative order and the concepts of minimum-phase behavior and zero dynamics. Then, formulas for input/output linearizing state feedback are derived, and the discrete-time GLC methodology is introduced. Three controllers within the GLC framework are formulated depending on the availability of process state measurements. The general nonlinear results are applied to linear systems, followed by a state-space reformulation of MAC. The linear controller (derived from the application of the GLC-error feedback structure to linear systems) and the state-space MAC are shown to be equivalent. A nonlinear model algorithmic controller is then developed and is shown to be identical to the GLC-error feedback controller. Finally, the three GLC structures are illustrated by a reactor example.

\section{Mathematical Preliminaries}

Consider SISO nonlinear processes described by a discretetime state-space model of the form:

$$
\left\{\begin{aligned}
x(k+1) & =\Phi[x(k), u(k)] \\
y(k) & =h[x(k)]
\end{aligned}\right.
$$

where $x$ denotes the vector of state variables, $u$ denotes the manipulated input, and $y$ represents an output (to be controlled), all in the form of deviation variables. It is assumed that $x \in X \subset \mathbb{R}^{n}$, and $u \in U \subset \mathbb{R}$, where $X$ and $U$ are openconnected sets that contain the origin (that is, the nominal equilibrium point). $\Phi(x, u)$ is an analytic vector function on $X \times U$, and $h(x)$ is an analytic scalar function on $X$.

The model of Eq. 1 can be viewed as the sampled-data representation of the continuous-time model:

$$
\left\{\begin{array}{l}
\dot{\bar{x}}(t)=f[\bar{x}(t)]+g[\bar{x}(t)] \bar{u}(t) \\
\bar{y}(t)=h[\bar{x}(t)]
\end{array}\right.
$$

that is, $x(k+1)=\Phi[x(k), u(k)]$ represents the solution at time $(k+1) \Delta t$ of the differential equations in Eq. 2 starting at time $k \Delta t$ in $\bar{x}(k \Delta t)=x(k)$ and with a constant $\bar{u}(t)=u(k)$, where $\Delta t$ is the sampling period.

The model of Eq. 1 is not affine in $u$, unlike the continuoustime model of Eq. 2. The sampled-data representation of an affine continuous-time model is nonaffine in general. Furthermore, as will be seen, even when the discrete-time model is affine, the input/output linearizing state feedback will be, in general, a nonaffine function of the external input (unlike in continuous-time case). Moreover, if a continuous-time nonlinear model has time delay, its sampled-data representation will still be in the form of Eq. 12.

A brief review of the notion of relative order (see Nijmeijer and van der Schaft, 1990 and references therein), which will be necessary for the subsequent developments, is given here.

Definition 1. For a system of the form of Eq. 1, the relative order of the output $y$ with respect to the manipulated input $u$ is the smallest integer $r$ for which

$$
\left[\frac{\partial h(x)}{\partial x}\right]\left[\frac{\partial \Phi(x, u)}{\partial x}\right]^{r-1}\left[\frac{\partial \Phi(x, u)}{\partial u}\right] \not \equiv 0
$$

If such an integer does not exist, we say that $r=\infty$.

Definition 1 implies that:

- If the relative order $r=1$, then

$$
\frac{\partial}{\partial u} h \circ \Phi \neq \equiv
$$

where $\circ$ denotes composition of functions that is, $h \circ \Phi \triangleq h[\Phi(x, u)]$.

- If the relative order $r=2$, then

$$
\frac{\partial}{\partial u} h \circ \Phi \equiv 0
$$

that is, $h \circ \Phi$ is a function of $x$ only, but

$$
\frac{\partial}{\partial u} h \circ \Phi \circ \Phi \neq 0
$$

- If the relative order $r=3$, then

$$
\frac{\partial}{\partial u} h \circ \Phi \equiv 0, \quad \frac{\partial}{\partial u} h \circ \Phi \circ \Phi \equiv 0
$$

that is, both $h \circ \Phi$ and $h \circ \Phi \circ \Phi$ are functions of $x$ only, but

$$
\frac{\partial}{\partial u} h \circ \Phi \circ \Phi \circ \Phi \neq 0
$$

- In general, the relative order $r$ is the smallest integer for which 


\section{$\frac{h \circ \Phi \circ \ldots \circ \Phi}{r \text { times }}$}

depends on $u$.

In view of this equivalent definition of the relative order $r$, the following notation will be used:

$$
\left\{\begin{array}{l}
h^{0}(x) \triangleq h(x) \\
h^{\ell}(x) \triangleq h^{\ell-1}[\Phi(x, u)], \quad \ell=1, \ldots, r-1,
\end{array}\right.
$$

In this notation,

$$
\begin{aligned}
\frac{\partial}{\partial u} h^{r-1}[\Phi(x, u)] \\
=\left[\frac{\partial h(x)}{\partial x}\right]\left[\frac{\partial \Phi(x, u)}{\partial x}\right]^{r-1}\left[\frac{\partial \Phi(x, u)}{\partial u}\right] \not \equiv 0 .
\end{aligned}
$$

Furthermore, the following relations will hold:

$$
\left\{\begin{array}{l}
y(k+\ell)=h^{p}[x(k)], \\
y(k+r)=h^{r-1}\{\Phi[x(k), u(k)]\}
\end{array} \quad \ell=0, \ldots, r-1\right.
$$

Therefore, $r$ is the smallest number of sampling periods after which the manipulated input move $u(k)$ affects the output $y$.

If the system output $y$ does not have a finite relative order $(r=\infty)$, this means that the manipulated input $u$ never affects the output $y$. In every well formulated control problem, the output $y$ must possess a finite relative order $r$.

In analogy to linear systems it is straightforward to show that the exact sampled-data representation of a dead-time-free SISO continuous-time system of the form of Eq. 2 with finite relative order $\bar{r}$ always has $r=1$ (Nijmeijer and van der Schaft, 1990). Thus, if a discrete-time nonlinear system of the form of Eq. 1 has $r>1,(r-1) \Delta t$ represents the plant dead-time, whereas the additional delay $\Delta t$ is the delay due to sampling. Because in discrete time, time delays simply increase the relative order, in this setting, time delay compensation is much easier than in continuous time.

Example 1. Consider the SISO discrete-time nonlinear system

$$
\left\{\begin{aligned}
x_{1}(k+1) & =x_{2}{ }^{2}(k)+x_{3}(k) \\
x_{2}(k+1) & =x_{1}(k) x_{2}{ }^{2}(k)+x_{3}(k) \\
x_{3}(k+1) & =u(k) \\
y(k) & =x_{1}(k)
\end{aligned}\right.
$$

For this system

$$
\begin{gathered}
\frac{\partial h[x(k)]}{\partial x}=\left[\begin{array}{lll}
1 & 0 & 0
\end{array}\right], \\
\frac{\partial \Phi[x(k), u(k)]}{\partial u}=\left[\begin{array}{l}
0 \\
0 \\
1
\end{array}\right],
\end{gathered}
$$

$$
\frac{\partial \Phi[x(k), u(k)]}{\partial x}=\left[\begin{array}{ccc}
0 & 2 x_{2}(k) & 1 \\
x_{2}^{2}(k) & 2 x_{1}(k) x_{2}(k) & 1 \\
0 & 0 & 0
\end{array}\right]
$$

and,

$$
\begin{gathered}
\frac{\partial h[x(k)]}{\partial x} \frac{\partial \Phi[x(k), u(k)]}{\partial u}=0, \\
\frac{\partial h[x(k)]}{\partial x} \frac{\partial \Phi[x(k), u(k)]}{\partial x} \frac{\partial \Phi[x(k), u(k)]}{\partial u}=1 \neq 0
\end{gathered}
$$

Therefore, $r=2$, and

$$
\begin{array}{rlrl}
h^{0}[x(k)] & =x_{1}(k) & =y(k) \\
h^{1}[x(k)] & =x_{2}{ }^{2}(k)+x_{3}(k) & =y(k+1) \\
h^{1}\{\Phi[x(k), u(k)]\} & =\left[x_{1}(k) x_{2}^{2}(k)+x_{3}(k)\right]^{2}+u(k)=y(k+2)
\end{array}
$$

which means that the present control move $u(k)$ affects the output after two sampling periods: the plant dead time is $\Delta t$ for this process.

For a process of the form of Eq. 1 with a finite relative order $r$, Eq. 3 implies that the algebraic equation

$$
h^{r-1}[\Phi(x, u)]=y
$$

is locally solvable in $u$ (via the implicit function theorem). The corresponding implicit function will be denoted by:

$$
u=\Psi_{o}(x, y)
$$

and will be assumed to be well-defined and unique on $X \times h(X)$.

\section{Minimum-phase behavior and zero dynamics}

The definition and interpretation of the relative order $r$ motivate the decomposition of the dynamic system of Eq. 1 into the subsystems in series: (i) a delay-free subsystem and (ii) a pure delay subsystem:

$$
\begin{aligned}
& \left\{\begin{aligned}
x(k+1) & =\Phi[x(k), u(k)] \\
y^{*}(k) & =h^{r-1}\{\Phi[x(k), u(k)]\} \quad \text { (delay-free) }
\end{aligned}\right. \\
& y(k+r)=y^{*}(k) \quad \text { (pure delay) }
\end{aligned}
$$

This decomposition is a direct generalization of the standard factorization of linear discrete-time systems into an invertible part (whose inverse is causal) and a pure delay. Furthermore, because of the solvability of Eq. 5, an inverse to the delayfree subsystem (Eq. 7) can be constructed:

$$
\left\{\begin{aligned}
x(k+1) & =\Phi\left[x(k), \Psi_{o}\left[x(k), y^{*}(k)\right]\right\} \\
u(k) & =\Psi_{o}\left[x(k), y^{*}(k)\right]
\end{aligned}\right.
$$

In the case of a linear system, the inverse system described by Eq. 9, has $(n-r)$ poles at the process zeros and $(r)$ poles at the origin. Consequently, stability of the inverse system is 
equivalent to finite process zeros being inside the unit circle. This motivates the following definition.

Definition 2. Given a discrete-time nonlinear system of the form of Eq. 1, its delay-free part is said to be minimum phase, if the dynamics

$$
x(k+1)=\Phi\left\{x(k), \Psi_{o}\left[x(k), y^{*}(k)\right]\right\}
$$

is locally asymptotically-stable. Otherwise, we will say that it is nonminimum phase.

The local asymptotic stability of the system of Eq. 10 can be checked, for example, via Lyapunov's first method, by calculating the eigenvalues of the Jacobian of the system evaluated at the equilibrium point. Using the definition of $\Psi_{o}$ (Eqs. 5 and 6) and the implicit function theorem, one can see that the Jacobian of the system of Eq. 10 at $\left(x_{o}, y_{o}\right)=(0,0)$ is equal to:

$$
\begin{aligned}
& \mathfrak{S}_{o}(x, u)=\left[\frac{\partial \Phi(x, u)}{\partial x}\right] \\
& \quad-\left[\frac{\partial \Phi(x, u)}{\partial u}\right]\left[\frac{\partial}{\partial x} h^{r-1}[\Phi(x, u)]\right]\left[\frac{\partial}{\partial u} h^{r-1}[\Phi(x, u)]\right]^{-1}
\end{aligned}
$$

evaluated at $(x, u)=(0,0)$. If all the eigenvalues of the Jacobian $I_{o}(x, u)$ are in the interior of the unit circle, the dynamics of Eq. 10 is guaranteed to be locally asymptotically-stable around the origin.

Definition 3. Given a discrete-time nonlinear system of the form Eq. 1, its delay-free part is said to be hyperbolically minimum phase, if all the eigenvalues of the Jacobian matrix $\mathfrak{S}_{o}(x, u)$, evaluated at the equilibrium point, are in the interior of the unit circle.

Remark 1. If the delay-free part of a discrete-time nonlinear system of the form of Eq. 1 is hyperbolically minimum phase, then it will also be minimum phase. However, the converse may not hold. The dynamics of Eq. 10 can have some eigenvalues on the unit circle and still be asymptotically-stable.

It is important to mention at this point that, in the systems theory literature (see, for example, Monaco and NormandCyrot, 1988), the notion of minimum-phase behavior, is defined via a notion of zero dynamics, which tries to generalize the continuous-time notion of zero dynamics of Byrnes and Isidori (1985). Note, however, that the associated normal form of discrete-time systems is built on the manifold:

$$
L^{*}=\left\{x \in X \mid h^{0}(x)=h^{1}(x)=\ldots=h^{r-1}(x)=0\right\}
$$

rather than the entire state space $X$. Consequently, the normal form of Monaco and Normand-Cyrot is not connected with the (forced) inverse dynamics, and its importance is mainly theoretical.

To obtain a discrete-time SISO analog of Byrnes-Isidori normal form, the following additional assumption must be made:

The vector fields in $\mathbb{R}^{n+1}$

$$
\left[\begin{array}{c}
\frac{\partial \Phi(x, u)}{\partial u} \\
---- \\
0
\end{array}\right] \text { and }\left[\begin{array}{c}
0 \\
\vdots \\
0 \\
--- \\
1
\end{array}\right]
$$

are involutive.

Under the above involutivity assumption, it is possible to find scalar functions $t_{j}(x), j=1, \ldots, n-r$ such that:

$$
\frac{\partial t_{j}[\Phi(x, u)]}{\partial u} \equiv 0
$$

and the coordinate transformation

$$
\zeta=\left[\begin{array}{c}
\zeta^{(0)} \\
-\zeta^{(1)}
\end{array}\right]=T(x)=\left[\begin{array}{c}
t_{1}(x) \\
\vdots \\
t_{n-r}(x) \\
-h^{0}(x) \\
h^{1}(x) \\
\vdots \\
h^{r-1}(x)
\end{array}\right]
$$

is locally invertible.

Under the above coordinate transformation, the system of Eq. 1 takes the normal form:

$$
\left\{\begin{aligned}
\zeta_{1}^{(0)}(k+1) & =F_{1}\left[\zeta^{(0)}(k), \zeta^{(1)}(k)\right] \\
\vdots & \\
\zeta_{n-r}^{(0)}(k+1) & =F_{n-r}\left[\zeta^{(0)}(k), \zeta^{(1)}(k)\right] \\
\zeta_{1}^{(1)}(k+1) & =\zeta_{2}^{(1)}(k) \\
\vdots & \\
\zeta_{r-1}^{(1)}(k+1) & =\zeta_{r}^{(1)}(k) \\
\zeta_{r}^{(1)}(k+1) & =\Omega\left[\zeta^{(0)}(k), \zeta^{(1)}(k), u(k)\right] \\
y(k) & =\zeta_{1}^{(1)}(k)
\end{aligned}\right.
$$

where

$$
\begin{aligned}
F_{j}(\zeta) & =t_{j}\left[\Phi\left(T^{-1}(\zeta), u\right)\right], j=1, \ldots, n-r \\
\Omega(\zeta, u) & =h^{r-1}\left\{\Phi\left[T^{-1}(\zeta), u\right]\right\}
\end{aligned}
$$

Remark 2. The previously mentioned involutivity condition is necessary and sufficient for the existence of $(n-1)$ linearly-independent solutions to the system of partial differential equations:

$$
\begin{gathered}
\sum_{i=1}^{n} \frac{\partial \Phi_{i}(x, u)}{\partial u} \frac{\partial w}{\partial x_{i}}=0 \\
\frac{\partial w}{\partial u}=0
\end{gathered}
$$

Alternatively stated, it is necessary and sufficient for the existence of $(n-1)$ linearly-independent functions of $x$ only, whose gradients are orthogonal to $[\partial \Phi(x, u)] / \partial u$ for every $u$. 
Consequently, it is necessary and sufficient for the existence of $(n-1)$ linearly-independent functions of $x$, whose composition with $\Phi(x, u)$ does not depend on $u$. If one denotes by $t_{j}(x), i=1, \ldots, n-1$ such functions, they will all be automatically linearly independent of $h^{r-1}(x)$. Furthermore, it is always possible to select $(n-r) t_{j}(x)$ 's so that they are also linearlyindependent of $h^{0}(x), \ldots, h^{r-2}(x)$; these will form the coordinate transformation of Eq. 12.

Remark 3. It is straightforward to show that the previously stated involutivity condition is equivalent to the following condition:

There exists a scalar function $S(x, u)$ such that:

$$
\left[\frac{\partial^{2} \Phi(x, u)}{\partial u^{2}}\right]=\S(x, u)\left[\frac{\partial \Phi(x, u)}{\partial u}\right]
$$

This is automatically satisfied for all $\Phi$ which are affine in $u$.

For a system in the above normal form (Eq. 13), the following relations hold:

$$
\zeta^{(1)}(k)=\left[\begin{array}{c}
y(k) \\
\vdots \\
y(k+r-1)
\end{array}\right]
$$

and

$$
\Omega\left[\zeta^{(0)}(k), \zeta^{(1)}(k), u(k)\right]=y(k+r)
$$

The normal form of Eq. 13, as well as its properties (Eqs. 14 and 15), are in complete analogy with the continuous-time normal form, where the time derivative operator $d / d t$ is now replaced by the forward shift operator. Thus, in analogy to the continuous-time case (see Kravaris, 1988), it is possible to define notions of both forced and unforced zero dynamics by considering the first $(n-r)$ equations of Eq. 13 as follows.

Definition 4. Consider a discrete-time nonlinear system of the form of Eq. 1 which has been transformed into the normal form of Eq. 13 via the coordinate transformation of Eq. 12. The dynamic system

$$
\left\{\begin{aligned}
\zeta_{1}^{(0)}(k+1) & =F_{1}\left[\zeta^{(0)}(k), \zeta^{(1)}(k)\right] \\
\vdots & \\
\zeta_{n-r}^{(0)}(k+1) & =F_{n-r}\left[\zeta^{(0)}(k), \zeta^{(1)}(k)\right]
\end{aligned}\right.
$$

with input $\zeta^{(1)}(k)$ is called the forced zero dynamics of the system described by Eq. 1. The dynamic system

$$
\left\{\begin{aligned}
\zeta_{1}^{(0)}(k+1) & =F_{1}\left[\zeta^{(0)}(k), 0\right] \\
\vdots & \\
\zeta_{n-r}^{(0)}(k+1)= & F_{n-r}\left[\zeta^{(0)}(k), 0\right]
\end{aligned}\right.
$$

is called the unforced zero dynamics or, simply, the zero dynamics of the system described by Eq. 1 .

The significance of the forced zero dynamics arises in connection with the inverse dynamics. The notion of (unforced) zero dynamics provides a nonlinear analog of the notion of zeros in linear systems. As mentioned earlier, the normal form of Monaco and Normand-Cyrot leads to a notion of unforced zero dynamics (which is, of course, equivalent to the one given in the above definition), but not to a notion of forced zero dynamics. On the other hand, the normal form of Eq. 13 is restricted to dynamic systems that satisfy the appropriate involutivity condition stated earlier.

A final comment must be made comparing the dynamics of the inverse of the delay-free part of the system (Eq. 9) to the zero dynamics. The poles of the linear approximation of the zero dynamics are exactly the $(n-r)$ zeros of the linear approximation of the process, whereas the linear approximation of Eq. 10 has $(r)$ additional poles at the origin. The latter do not affect the stability properties of Eq. 10 and, for this reason, the system of Eq. 10 is suitable for defining minimum-phase behavior. However, it is not appropriate to refer to the dynamics of Eq. 10 as the "zero dynamics" because of its additional poles at the origin.

Example 2. To illustrate the above concepts, the system described by Eq. 4 is considered. For this example, the delayfree and pure delay subsystems are:

$$
\left\{\begin{aligned}
& x_{1}(k+1)=x_{2}^{2}(k)+x_{3}(k) \\
& x_{2}(k+1)=x_{1}(k) x_{2}^{2}(k)+x_{3}(k) \\
& x_{3}(k+1)=u(k) \\
& y^{*}(k)= {\left[x_{1}(k) x_{2}^{2}(k)+x_{3}(k)\right]^{2}+u(k) } \\
& y(k+2)=y^{*}(k) \quad \text { (pure delay-free) }
\end{aligned}\right.
$$

The inverse of the delay-free subsystem is:

$$
\left\{\begin{aligned}
x_{1}(k+1) & =x_{2}^{2}(k)+x_{3}(k) \\
x_{2}(k+1) & =x_{1}(k) x_{2}^{2}(k)+x_{3}(k) \\
x_{3}(k+1) & =y^{*}(k)-\left[x_{1}(k) x_{2}^{2}(k)+x_{3}(k)\right]^{2} \\
u(k) & =y^{*}(k)-\left[x_{1}(k) x_{2}^{2}(k)+x_{3}(k)\right]^{2}
\end{aligned}\right.
$$

The Jacobian of the closed-loop system, evaluated at the equilibrium point $\left(x_{e}, u_{e}\right)=(0,0,0,0)$, is:

$$
\mathfrak{I}_{o}(x, u)=\left[\begin{array}{lll}
0 & 0 & 1 \\
0 & 0 & 1 \\
0 & 0 & 0
\end{array}\right]
$$

which has three eigenvalues at the origin $\left(\lambda_{i}=0, i=1,2,3\right)$, therefore, the delay-free part of the system is hyperbolically minimum phase. Furthermore, the locally invertible coordinate transformation

$$
\zeta=\left[\begin{array}{c}
\zeta_{1}^{(0)} \\
\zeta_{1}^{(1)} \\
\zeta_{2}^{(1)}
\end{array}\right]=T(x)=\left[\begin{array}{c}
x_{2} \\
x_{1} \\
x_{2}{ }^{2}+x_{3}
\end{array}\right]
$$

transforms the system of Eq. 4 into normal form

$$
\left\{\begin{aligned}
\zeta_{1}^{(0)}(k+1) & =\zeta_{1}^{(1)}(k) \zeta_{1}^{(0)^{2}}(k)+\zeta_{2}^{(1)}(k)-\zeta_{1}^{(0)^{2}}(k) \\
\zeta_{1}^{(1)}(k+1) & =\zeta_{2}^{(1)}(k) \\
\zeta_{2}^{(1)}(k+1) & =\left[\zeta_{1}^{(1)}(k) \zeta_{1}^{(0)^{2}}(k)+\zeta_{2}^{(1)}(k)-\zeta_{1}^{(0)^{2}}(k)\right]^{2}+u(k) \\
y(k) & =\zeta_{1}^{(1)}(k)
\end{aligned}\right.
$$


The corresponding forced and unforced zero dynamics, repspectively, are given by:

$$
\zeta_{1}^{(0)}(k+1)=\zeta_{1}^{(1)}(k) \zeta_{1}^{(0)^{2}}(k)+\zeta_{2}^{(1)}(k)-\zeta_{1}^{(0)^{2}}(k)
$$

and

$$
\zeta_{1}^{(0)}(k+1)=-\zeta_{1}^{(0)^{2}}(k)
$$

Clearly, the zero dynamics is locally stable (its linear approximation around the origin has a pole at zero), which confirm the previous results obtained from the eigenvalues of the $\mathrm{Ja}$ cobian matrix $\mathfrak{I}_{o}(x, u)$.

\section{Input/Output Linearizing Feedback}

In this section, a discrete-time analog of input/output linearizing feedback is developed by extending available theoretical results on output dead-beat control of nonlinear systems (Glad, 1987; Monaco et al., 1989).

The results of this section will play an instrumental role in the development of general controller synthesis strategies for discrete-time nonlinear systems.

In analogy to continuous-time nonlinear systems, one can pose the problem of synthesizing a static state feedback of the form:

$$
u(k)=\Psi[x(k), v(k)]
$$

with $\{\partial \Psi[x(k), v(k)]\} / \partial v \neq 0$, which, when applied to a dynamic system of the form of Eq. 1, induces a linear closedloop input/output behavior.

It is straightforward to show that the closed-loop system resulting from the feedback of Eq. 18:

$$
\left\{\begin{aligned}
x(k+1) & =\Phi\{x(k), \Psi[x(k), v(k)]\} \\
y(k) & =h[x(k)]
\end{aligned}\right.
$$

has the same relative order as the open-loop system (Eq. 1). Consequently, if $r$ is the relative order of the open-loop system, a closed-loop dynamics with relative order $r$ must be requested. If linear closed-loop input/output dynamics of minimal order is desirable, this has the general form:

$$
y(k+r)+\sum_{\ell=1}^{r} \beta_{\ell} y(k+r-\ell)=\beta_{0} v(k)
$$

where $\beta_{\ell}, \ell=0, \ldots, r$ are constant scalars with $\beta_{0} \neq 0$. Equivalently, the input/output dynamics (Eq. 19) can be represented by a $z$-domain transfer function as:

$$
y(z)=G_{i}(z) v(z)
$$

where

$$
\begin{aligned}
G_{i}(z)=\frac{\beta_{0}}{z^{r}+\beta_{1} z^{r-1}+\ldots+\beta_{r-1} z+\beta_{r}} & \\
& =z^{-r} \frac{\beta_{0}}{1+\beta_{1} z^{-1}+\ldots+\beta_{r-1} z^{1-r}+\beta_{r} z^{-r}}
\end{aligned}
$$

which has $r$ poles at the roots of the characteristic equation:

$$
z^{r}+\beta_{1} z^{r-1}+\ldots+\beta_{r-1} z+\beta_{r}=0
$$

and no zeros. Therefore, the order of the closed-loop system is $r$.

The necessary static state feedback that induces the closedloop input/output dynamics of Eq. 19 can be calculated from the definition and properties of the relative order $r$. It is the implicit function defined as the solution for $u(k)$ of the algebraic equation:

$$
h^{r-1}\{\Phi[x(k), u(k)]\}=\beta_{0} v(k)-\sum_{\ell=1}^{r} \beta_{\ell} h^{r-\ell}[x(k)]
$$

Recalling the definition of the function $\Psi_{o}$ (Eqs. 5 and 6), this can be written as:

$$
u(k)=\Psi_{o}\left\{x(k), \beta_{0} v(k)-\sum_{\ell=1}^{r} \beta_{p} h^{r-\ell}[x(k)]\right\}
$$

Note that in the special case $\beta_{0}=1, \beta_{\ell}=0, \ell=1, \ldots, r$, the state feedback of Eq. 22 becomes:

$$
u(k)=\Psi_{o}[x(k), v(k)]
$$

and the resulting input/output behavior is:

$$
y(k+r)=v(k)
$$

which is an output dead-beat response. Although from practical standpoint, the dead-beat response is not a good choice (in terms of its robustness properties), dead-beat control has considerable theoretical importance, which attracted a lot of attention in the systems theory literature (for example, O'Reilly, 1981; Glad, 1987; Isermann, 1989). The closed-loop response of Eq. 24 is the fastest achievable closed-loop response in terms of tracking the reference input $v$. Also, the closed-loop dynamics arising from the feedback of Eq. 23:

$$
x(k+1)=\Phi\left\{x(k), \Psi_{o}[x(k), v(k)]\right\}
$$

is identical to the dynamics of the inverse of the delay-free part of the process (cf. Eq. 9). Therefore, the delay-free part of a system of the form of Eq. 1 is minimum-phase if and only if the closed-loop dynamics associated with output deadbeat response is asymptotically stable (Glad, 1987; Monaco and Normand-Cyrot, 1988).

For practical purposes, one must use $\beta_{\ell} \neq 0$ to obtain a sufficiently robust response. For this reason, one must analyze the closed-loop system arising from the general state feedback law of Eq. 22:

$$
\left\{\begin{aligned}
x(k+1) & =\Phi\left[x(k), \Psi_{o}\left(x(k), \beta_{0} v(k)-\sum_{\ell=1}^{r} \beta_{\ell} h^{r-p}[x(k)]\right)\right] \\
y(k) & =h[x(k)]
\end{aligned}\right.
$$

The input/output behavior of Eq. 25 is, of course, governed 
by the transfer function of Eq. 20, and therefore, the input/ output closed-loop stability and performance characteristics are determined by the roots of the characteristic equation (Eq. 21). The asymptotic stability characteristics of the dynamics of Eq. 25 depend on the eigenvalues of the Jacobian of the system, evaluated at the equilibrium point. Using the definition of $\Psi_{o}$ (Eqs. 5 and 6) and the implicit function theorem, the Jacobian of the system of Eq. 25 is given by:

$$
\begin{array}{r}
\mathfrak{J}_{\beta}(x, u)=\left[\frac{\partial \Phi(x, u)}{\partial x}\right]-\left[\frac{\partial \Phi(x, u)}{\partial u}\right]\left\{\frac{\partial}{\partial x} h^{r-1}[\Phi(x, u)]\right. \\
\left.+\sum_{\ell=1}^{r} \beta_{\ell} \frac{\partial h^{r-\ell}(x)}{\partial x}\right\}\left\{\frac{\partial}{\partial u} h^{r-1}[\Phi(x, u)]\right\}^{-1}
\end{array}
$$

Thus, if the eigenvalues of $\mathcal{G}_{\beta}(0,0)$ are in the interior of the unit circle, the closed-loop system of Eq. 25 is guaranteed to be locally internally-stable. Precise conditions are given in the proposition that follows:

Proposition 1. Consider a system of the form of Eq. 1 with finite relative order $r$, which is subject to a state feedback of the form of $E q .22$, where the parameters $\beta_{b} \ell=1, \ldots, r$ are chosen so that the roots of the characteristic equation (Eq. 21) are in the interior of the unit circle. If the delay-free part of the system of Eq. 1 is hyperbolically minimum phase, then the closed-loop system (Eq. 25) is locally asymptotically stable. (The proof is given in the Appendix.)

As a consequence of the above proposition, the input/output linearizing state feedback (Eq. 22) can form the basis for controller design for all hyperbolically minimum-phase systems of the general form of Eq. 1.

Example 3. Consider the system given in Example 1 (Eq. 4). The corresponding input/output linearizing feedback is:

$$
\begin{array}{r}
u(k)=\beta_{0} v(k)-\beta_{2} x_{1}(k)-\beta_{1}\left[x_{2}^{2}(k)+x_{3}(k)\right] \\
-\left[x_{1}(k) x_{2}^{2}(k)+x_{3}(k)\right]^{2}
\end{array}
$$

which induces the closed-loop response

$$
y(k+2)+\beta_{1} y(k+1)+\beta_{2} y(k)=\beta_{0} v(k)
$$

with $\beta_{0} \neq 0$, or, in the $z$-domain,

$$
y(z)=\frac{\beta_{0}}{z^{2}+\beta_{1} z+\beta_{2}} v(z)
$$

\section{State feedback simplifications}

The number of adjustable parameters can be reduced by making decisions on the form of the desirable input/output behavior of the closed-loop system, particularly unity static gain in the $v-y$ system and the first-order-plus-deadtime response.

Unity Static Gain in the v-y System. The requirement of unity static gain in the closed-loop $v-y$ system translates into:

$$
G_{i}(1)=1
$$

or equivalently,

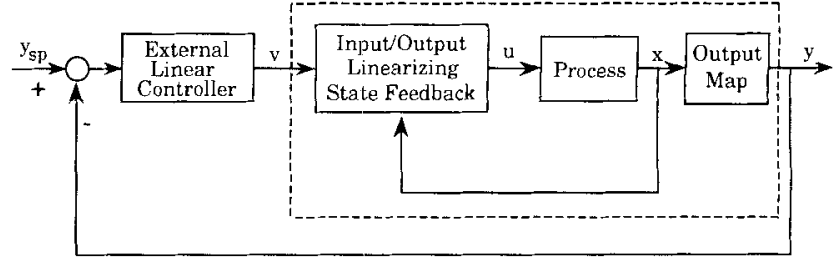

Figure 1. GLC structure.

$$
1+\sum_{\ell=1}^{r} \beta_{p}=\beta_{0}
$$

As will be seen later, this condition does not need to be imposed at this stage, because the external controller, which will be synthesized later, guarantees offsetless tracking of the set point.

First-Order-Plus-Dead-time Response. A popular type of requested closed-loop response is one of the "first-order-plusdeadtime." This can be achieved by setting:

$$
\beta_{\ell}=0, \quad \ell=2, \ldots, r
$$

In this case, the closed-loop response further simplifies into:

$$
y(k+r)+\beta_{1} y(k+r-1)=\beta_{0} v(k)
$$

or in the $z$-domain,

$$
\frac{y(z)}{v(z)}=z^{-r} \frac{\beta_{0}}{1+\beta_{1} z^{-1}}
$$

If, in addition, unity static gain is desirable, the condition

$$
1+\beta_{1}=\beta_{0}
$$

must hold, leading to:

$$
\frac{y(z)}{v(z)}=z^{-r} \frac{1+\beta_{1}}{1+\beta_{1} z^{-1}}
$$

\section{Globally Linearizing Control Structure}

Once a nonlinear system is linearized in an input/output sense via the state feedback of Eq. 22, one can place a linear controller with integral action around the linear $v-y$ system for offsetless tracking of set points in the presence of modeling errors and process disturbances. This leads to the GLC structure, which is depicted in Figure 1. The GLC design methodology was developed for continuous-time systems in Kravaris and Chung (1987) and Kravaris and Soroush (1990). In this and subsequent sections, the development will be completely parallel to the continuous-time GLC, considering general statespace discrete-time models of the form of Eq. 1, which are input/output linearized by the static state feedback of Eq. 22 .

\section{External controller}

As shown earlier, under the state feedback of Eq. 22, the closed-loop input/output behavior of the system is linear and is given by: 


$$
y(z)=G_{i}(z) v(z)
$$

where

$$
G_{i}(z)=\frac{\beta_{0}}{z^{r}+\beta_{1} z^{r-1}+\ldots+\beta_{r-1} z+\beta_{r}}
$$

(even for $r>1$ ), although in general, one must sacrifice performance for the sake of simplicity of the external controller.

For the purpose of on-line implementation of the external controller $G_{e}(z)$ defined by Eqs. 29 and 30, a state space realization must be constructed. One can readily obtain a minimal-order state-space realization (Isermann, 1989):

$$
\begin{aligned}
\xi_{1}(k+1) & =-\gamma_{1} \xi_{1}(k)-\cdots-\gamma_{r-1} \xi_{r-1}(k)+\left(1+\gamma_{1}+\cdots+\gamma_{r-1}\right) \xi_{r}(k)+\left(1+\gamma_{1}+\cdots+\gamma_{r}\right) e(k) \\
\xi_{2}(k+1) & =\xi_{1}(k) \\
& \vdots \\
\xi_{r}(k+1) & =\xi_{r-1}(k) \\
v(k) & =\frac{\left(\beta_{1}-\gamma_{1}\right) \xi_{1}(k)+\cdots+\left(\beta_{r-1}-\gamma_{r-1}\right) \xi_{r-1}(k)+\left(1+\gamma_{1}+\cdots+\gamma_{r-1}+\beta_{r}\right) \xi_{r}(k)}{\beta_{0}}+\frac{\left(1+\gamma_{1}+\cdots+\gamma_{r-1}+\gamma_{r}\right) e(k)}{\beta_{0}}
\end{aligned}
$$

Once an overall closed-loop behavior of the general form

$$
y(z)=G_{o}(z) y_{s p}(z), \text { such that } G_{o}(1)=1
$$

where $y_{s p}$ is the output set point, is specified, and then the problem of synthesizing the necessary linear external controller of the GLC structure $G_{e}(z)$ becomes straightforward:

$$
G_{e}(z)=\frac{G_{o}(z)}{G_{i}(z)\left[1-G_{o}(z)\right]}
$$

Note that the condition $G_{o}(1)=1$ (in Eq. 27), which guarantees the offsetless tracking of the set point, induces integral action in the synthesized external controller $G_{e}(z)$.

In particular, if an overall closed-loop behavior of the form:

$$
G_{o}(z)=\frac{R(1)}{R(z)}
$$

is desirable, where $R(z)$ is a polynomial in $z$ with $R(1)$ nonzero and finite, the external linear controller $G_{e}(z)$ is given by:

$$
G_{e}(z)=\left(\frac{R(1)}{\beta_{0}}\right)\left(\frac{z^{r}+\beta_{1} z^{r-1}+\ldots+\beta_{r-1} z+\beta_{r}}{R(z)-R(1)}\right)
$$

Causality of the external controller $G_{e}(z)$ is guaranteed as long as the polynomial $R(z)$ is chosen to be of degree $r$ :

$$
R(z)=z^{r}+\gamma_{1} z^{r-1}+\ldots+\gamma_{r-1} z+\gamma_{r}
$$

where $\gamma_{\ell}, \ell=1, \ldots, r$ are adjustable scalar parameters.

Remark 4. It is interesting to observe that for a dead-timefree process $(r=1)$, the external controller $G_{e}(z)$, defined by Eqs. 29 and 30, becomes:

$$
G_{e}(z)=\left(\frac{1+\gamma_{1}}{\beta_{0}}\right)\left(\frac{z+\beta_{1}}{z-1}\right)
$$

which is exactly a digital PI controller. The use of PI controllers to control a $v-y$ system may be sufficient in many applications or in a more compact notation,

$$
\begin{gathered}
\xi(k+1)=A_{c} \xi(k)+B_{c} e(k) \\
\beta_{0} v(k)=C_{c} \xi(k)+D_{c} e(k)
\end{gathered}
$$

where

$$
\xi(k)=\left[\begin{array}{c}
\xi_{1}(k) \\
\vdots \\
\xi_{r}(k)
\end{array}\right],
$$

and

$$
\begin{gathered}
A_{c}=\left[\begin{array}{ccccc}
-\gamma_{1} & -\gamma_{2} & \cdots & -\gamma_{r-1} & \left(1+\gamma_{1}+\cdots+\gamma_{r-1}\right) \\
1 & 0 & \cdots & 0 & 0 \\
0 & 1 & \cdots & 0 & 0 \\
\vdots & \vdots & & \vdots & \vdots \\
0 & 0 & \cdots & 1 & 0
\end{array}\right] \\
B_{c}=\left[\begin{array}{c}
1+\gamma_{1}+\cdots+\gamma_{r} \\
0 \\
0 \\
\vdots \\
0
\end{array}\right] \\
C_{r}=\left[\left(\beta_{1}-\gamma_{1}\right)\left(\beta_{2}-\gamma_{2}\right) \cdots\left(\beta_{r-1}-\gamma_{r-1}\right)\right. \\
D_{c}=1+\gamma_{1}+\cdots+\gamma_{r-1}+\gamma_{r}
\end{gathered}
$$

Note that both the order of the external controller transfer function and the order of its state-space realization (Eq. 31) are $r$; hence, the realization of Eq. 31 is minimal.

Remark 5. When $r=1$ (dead-time-free process), the realization of Eq. 31 simplifies into: 


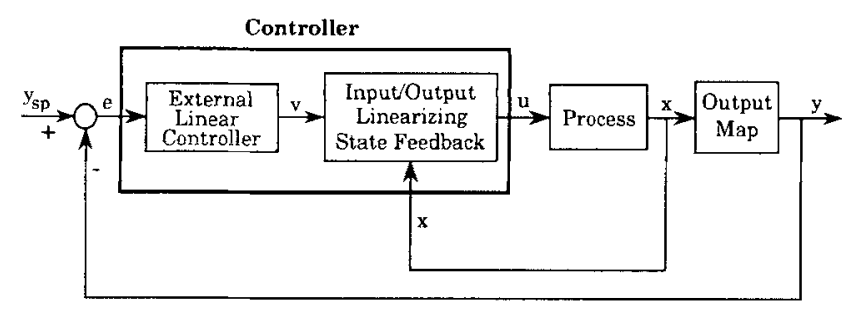

Figure 2a. GLC-state-and-error-feedback structure ("basic" GLC).

$$
\begin{aligned}
\xi_{1}(k+1) & =\xi_{1}(k)+\left(1+\gamma_{1}\right) e(k) \\
v(k) & =\frac{1+\beta_{1}}{\beta_{0}} \xi_{1}(k)+\frac{1+\gamma_{1}}{\beta_{0}} e(k)
\end{aligned}
$$

which is a realization of a digital PI controller. This corresponds to $A_{c}=1, B_{c}=1+\gamma_{1}, C_{c}=1+\beta_{1}, D_{c}=1+\gamma_{1}$ (all scalar).

\section{Overall controller (mixed error and state feedback)}

In the case that all the process state variables are measured on-line, the state feedback (Eq. 22) and the external controller (Eq. 31) are implemented to induce a desired linear overall closed-loop response.

Theorem 1. Consider the nonlinear process described by Eq. 1 with finite relative order $r$. Then the dynamic system

$$
\left\{\begin{aligned}
\xi(k+1) & =A_{c} \xi(k)+B_{c} e(k) \\
u(k) & =\Psi_{o}\left(x(k), C_{c} \xi(k)+D_{c} e(k)-\sum_{\ell=1}^{r} \beta_{\ell} h^{r-\ell}[x(k)]\right)
\end{aligned}\right.
$$

where $\Psi_{o}(.,$.$) is defined by Eqs. 5$ and 6, and $A_{c}, B_{c}, C_{c}$ and $D_{c}$ are the system matrices of the realization of $E q .31$, represents an rth order state-space realization of a mixed errorand state-feedback controller that induces the closed-loop input/output behavior described by Eqs. 29 and 30:

$$
y(k+r)+\sum_{\ell=1}^{r} \gamma_{\ell} y(k+r-\ell)=\left(1+\sum_{\ell=1}^{r} \gamma_{\ell}\right) y_{s p}(k)
$$

where $\gamma \ell, \ell=1, \ldots, r$ are adjustable parameters and $y_{s p}$ is the output set point. (The proof is given in the Appendix.)

The block diagram of the mixed error- and state-feedback controller (Eq. 32) is depicted in Figure 2a. This controller structure will be referred to as the "Basic" GLC structure.

\section{Dynamic Output Feedback Synthesis via GLC} Formulation of the dynamic output feedback synthesis problem

The GLC structure presented in the previous section results in an overall feedback controller which is mixed error and state feedback. In many practical cases, on-line measurement of all the states is either infeasible or too expensive. In a typical situation, only the output is measured on-line, and this motivates the problem of synthesizing nonlinear output feedback control laws for accurate tracking of set points and effective rejection of disturbances and modeling errors.

Unlike the state feedback law of Eq. 22, which was static, in an output feedback setting one must seek for a dynamic feedback law to achieve satisfactory servo and regulatory behavior in the closed loop. Furthermore, because there is no nonlinear analog of a transfer function to represent the input/ output dynamics of a nonlinear system, the dynamic output feedback controller must be represented in terms of a statespace realization. For this reason, the formulation and solution of the controller synthesis problem must be done in state space.

In view of the above considerations, the following synthesis problem is posed: Given a nonlinear process described by a state-space model of the form of Eq. 1, calculate a state-space realization of a nonlinear dynamic output feedback controller which induces a closed-loop input/output behavior of the form of Eq. 33.

The posed synthesis problem can be conveniently attacked within the GLC framework, if the input/output linearizing feedback is combined with an appropriate state observer. Since there is no separation principle in nonlinear control, the state feedback/state observer combination will be used in deriving synthesis formulas (state-space controller realizations), although the analysis of the closed-loop behavior must be performed by viewing the resulting controller as one entity, without attempting to "superimpose" the behavior of the state feedback and the state observer. The approach, which is followed in this section, is completely parallel to the continuous-time output feedback synthesis approach in Daoutidis and Kravaris (1992).

\section{Synthesis of error-feedback controllers: GLC-error feed-} back structure

When the process model of Eq. 1 is open-loop-stable, one can use an open-loop observer to reconstruct the process states; this motivates on-line simulation of the process model:

$$
w(k+1)=\Phi[w(k), u(k)]
$$

The state estimates $w$ can be used in the input/output linearizing state feedback:

$$
u(k)=\Psi_{o}\left(w(k), \beta_{0} v(k)-\sum_{\ell=1}^{r} \beta_{\ell} h^{r-\ell}[w(k)]\right)
$$

where $v$ is obtained from the external controller of Eq. 31 . Combining Eqs. 31, 34 and 35, we obtain a state-space realization of a controller, which induces the requested closedloop dynamics (Eq. 33). This is established in the theorem that follows.

Theorem 2. Consider the nonlinear process described by Eq. 1 with finite relative order $r$. Then the dynamic system: 


$$
\left\{\begin{aligned}
& \xi(k+1)=A_{c} \xi(k)+B_{c} e(k) \\
& w(k+1)=\Phi\left(w(k), \Psi_{o}\left(w(k), C_{c} \xi(k)+D_{c} e(k)\right.\right. \\
&\left.\left.-\sum_{\ell=1}^{r} \beta_{\ell} h^{r-\ell}[w(k)]\right)\right) \\
& u(k)=\Psi_{o}\left(w(k), C_{c} \xi(k)+D_{c} e(k)\right. \\
&\left.-\sum_{\ell=1}^{r} \beta_{\ell} h^{r-1}[w(k)]\right)
\end{aligned}\right.
$$

where $\Psi_{o}(.,$.$) is defined by Eqs. 5$ and 6 , and $A_{c}, B_{c}, C_{c}$ and $D_{c}$ are the system matrices of the realization of $E q .31$, represents an $(n+r)$ th order state-space realization of a dynamic output feedback controller that induces the closed-loop input/ error behavior of Eq. 33. (The proof is given in the Appendix.)

Corollary (Reduced-Order Realization). Under the assumptions of Theorem 2, the dynamic system:

$$
\left\{\begin{aligned}
w(k+1)= & \Phi\left(w(k), \Psi_{o}\left(w(k),-\sum_{\ell=1}^{r-1} \gamma_{\ell}\left[h^{r-\ell}[w(k)]\right.\right.\right. \\
& \left.\left.-h[w(k)]]+h[w(k)]+\left(1+\sum_{\ell=1}^{r} \gamma_{\ell}\right) e(k)\right)\right) \\
u(k)= & \Psi_{o}\left(w(k),-\sum_{\ell=1}^{r-1} \gamma_{\ell}\left[h^{r-\ell}[w(k)]-h[w(k)]\right]\right. \\
& \left.+h[w(k)]+\left(1+\sum_{\ell=1}^{r} \gamma_{\ell}\right) e(k)\right)
\end{aligned}\right.
$$

represents an nth order state-space realization of a dynamic error feedback controller that induces the closed-loop input/ output behavior of Eq. 33. (The proof is given in the Appendix.)

Remark 6. The controller realizations (Eqs. 36 and 37) represent a dynamic error-feedback controller (the only input to the controller is the error) with integral action. The overall control structure as well as the various parts of the controller (Eq. 36) are shown in Figure 2b. This controller structure will be referred to as the GLC-error-feedback structure.

Remark 7. The controller realization of Eq. 37 can be interpreted as a feedforward controller that induces the errorto-output dynamics:

$$
\begin{aligned}
& {[y(k+r)-y(k)]+\sum_{\ell=1}^{r} \gamma_{\ell}[y(k+r-\ell)-y(k)]} \\
& \qquad=\left(1+\sum_{\ell=1}^{r} \gamma_{\ell}\right) e(k)
\end{aligned}
$$

This interpretation suggests an alternative way of deriving the controller of Eq. 37.

\section{Some practical considerations: controller simplification}

From a design point of view, it makes sense to try to reduce the number of adjustable parameters as long as it gives enough

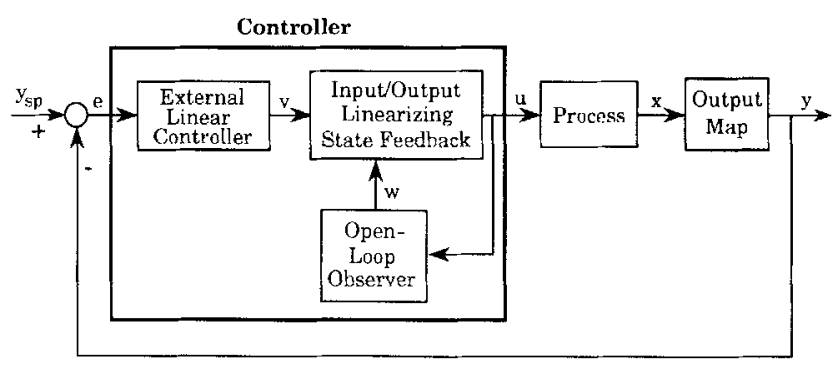

Figure 2b. GLC-error feedback structure.

flexibility to "shape" the closed-loop response. One way of doing this is by placing $(r-1)$ poles at the origin and leaving the remaining pole adjustable. This corresponds to choosing:

$$
\left\{\begin{aligned}
\gamma_{1} & =-\alpha, \quad 0<\alpha<1 \\
\gamma_{2} & =0 \\
& \vdots \\
\gamma_{r} & =0
\end{aligned}\right.
$$

that is, a desirable closed-loop input/output behavior

$$
y(k+r)-\alpha y(k+r-1)=(1-\alpha) y_{s p}(k)
$$

or

$$
\frac{y(z)}{y_{s p}(z)}=z^{-r} \frac{1-\alpha}{1-\alpha z^{-1}}
$$

which is first-order-plus-dead-time. This makes intuitive sense, because the dead time is preserved in the closed loop, and also the first order is the simplest type of response. Furthermore,

$$
\frac{1-\alpha}{1-\alpha z^{-1}}
$$

is the transfer function of a first-order filter; therefore, the controller that leads to such a response can find a nice IMC interpretation in linear systems (see original IMC article by Garcia and Morari, 1982). Finally, practical experience from linear systems has shown that although this kind of response has only one tunable parameter, it gives enough flexibility to the designer.

When the parameters $\gamma_{l}$ are chosen according to Eq. 38, the controller of Eq. 37 simplifies into:

$$
\left\{\begin{aligned}
w(k+1)=\Phi[w(k), & \Psi_{o}\left[w(k), \alpha h^{r-1}[w(k)]\right. \\
& +(1-\alpha) h[w(k)]+(1-\alpha) e(k)]\} \\
u(k)=\Psi_{o}\left\{w(k), \alpha h^{r-1}[w(k)]+(1-\alpha) h[w(k)]\right. & +(1-\alpha) e(k)\}
\end{aligned}\right.
$$

Remark 8. It is straightforward to derive a Smith predictor interpretation/implementation of the controller of Eq. 39 when $r>1$, along the lines of the linear discrete-time Smith predictor 
of Alevisakis and Seborg (1973). The Smith predictor will try to predict the auxiliary output $\mathrm{y}^{\otimes}=h^{r-1}(x)$, which represents the process output when the process dead time $(r-1) \Delta t$ is "factored out." This can be done by simulating:

$$
\begin{aligned}
& (\mathfrak{X}(k+1)=\Phi[\mathfrak{X}(k), u(k)], \quad \text { (Process model) } \\
& \delta y^{\otimes}(k)=h^{r-1}[X(k)]-h[\mathscr{X}(k)], \quad \text { (Corrective Signal) } \\
& \hat{y}^{\otimes}(k)=y(k)+\delta y^{\otimes}(k), \quad \text { (Prediction of dead-time- } \\
& \text { free output) }
\end{aligned}
$$

The prediction is compared to the set point and the error:

$$
e^{\otimes}(k)=y_{s p}(k)-\hat{y}^{\otimes}(k)
$$

is fed to a GLC-error-feedback controller, synthesized for the dead-time-free output $y^{\otimes}=h^{r-1}(x)$ :

$$
\left\{\begin{array}{c}
w(k+1)=\Phi\left\{w(k), \Psi_{o}\left[w(k), h^{r-1}[w(k)]+(1-\alpha) e^{\otimes}(k)\right]\right\} \\
u(k)=\Psi_{o}\left\{w(k), h^{r-1}[w(k)]+(1-\alpha) e^{\otimes}(k)\right\}
\end{array}\right.
$$

It is understood that the states $\mathscr{X}$ of the Smith predictor and the states $w$ of the above GLC controller will be consistently initialized $[X(0)=w(0)]$, in which case $X(k)=w(k)$ for all $k$; this makes the Smith predictor structure implementation equivalent to the implementation suggested by Eq. 39. It must be noted, however, that in discrete time, the motivation/meaningfulness of the Smith predictor concept is not as strong as in continuous time.

Synthesis of output feedback controllers for dead-timefree processes: GLC-two degree of freedom output feedback structure

The approach developed in the previous subsection can be applied only to open-loop stable processes whose delay-free part is minimum phase. In the case of open-loop instability, any error in the observer initialization would grow indefinitely, leading to obvious internal stability problems. However, for the case of dead-time-free minimum-phase processes, the normal form representation (Eq. 13) suggests an alternative way of state reconstruction, valid even in the presence of possible open-loop instability.

Consider the normal form representation (Eq. 13) for $r=1$ (dead-time-free process):

$$
\begin{aligned}
\zeta_{1}^{(0)}(k+1) & =F_{1}\left[\zeta_{1}^{(0)}(k), \ldots, \zeta_{n-1}^{(0)}(k), \zeta_{1}^{(1)}(k)\right] \\
& \vdots \\
\zeta_{n-1}^{(0)}(k+1) & =F_{n-1}\left[\zeta_{1}^{(0)}(k), \ldots, \zeta_{n-1}^{(0)}(k), \zeta_{1}^{(1)}(k)\right] \\
\zeta_{1}^{(1)}(k+1) & =\Omega\left[\zeta_{1}^{(0)}(k), \ldots, \zeta_{n-1}^{(0)}(k), \zeta_{1}^{(1)}(k), u(k)\right] \\
y(k) & =\zeta_{1}^{(1)}(k)
\end{aligned}
$$

We see from (Eq. 40) that the last state is exactly the measured output whereas the first $(n-1)$ states can be reconstructed by simulating the first $(n-1)$ state equations (the forced zero dynamics), driven by the output measurement. This leads to the following reduced-order observer:

$$
\begin{aligned}
\eta_{1}(k+1) & =F_{1}[\eta(k), y(k)] \\
& \vdots \\
\eta_{n-1}(k+1) & =F_{n-1}[\eta(k), y(k)]
\end{aligned}
$$

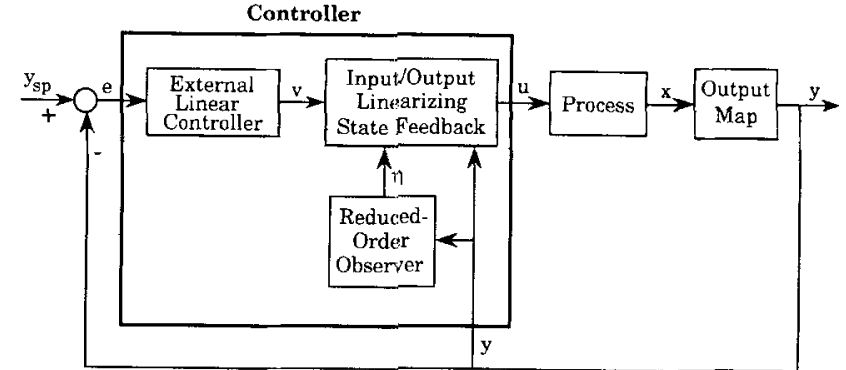

Figure 2c. GLC-two degree of freedom output feedback structure.

where $\eta=\zeta^{(0)}$, or, in a compact form $\eta(k+1)=F[\eta(k), y(k)]$. The state estimates $\eta$ can now be used in the input/output linearizing feedback (Eq. 22) which, combined with an external controller, leads to an overall output feedback controller for the process. This is established in the theorem that follows:

Theorem 3. Consider the nonlinear process described by Eq. 40. Then the dynamic system:

$$
\left\{\begin{aligned}
\xi(k+1) & =\xi(k)+\left(1+\gamma_{1}\right) e(k) \\
\eta(k+1) & =F[\eta(k), y(k)] \\
u(k) & =\Psi_{o}\left(\left[\begin{array}{c}
\eta(k) \\
y(k)
\end{array}\right], \beta_{1}[\xi(k)-y(k)]+\xi(k)+\left(1+\gamma_{1}\right) e(k)\right)
\end{aligned}\right.
$$

represents an nth order state-space realization of a dynamic output feedback controller that induces the closed-loop input/ output dynamics:

$$
y(k+1)+\gamma_{1} y(k)=\left(1+\gamma_{1}\right) y_{s p}(k)
$$

(The proof is given in the Appendix.)

Remark 9. The controller realization of Eq. 42 is a nonlinear analog of a two-degree-of-freedom controller: a mixed error- and output-feedback controller. This is consistent with the intuition from linear systems theory where a two-degreeof-freedom controller is usually employed for open-loop unstable systems, with the output feedback having a stabilizing effect on the overall control action. The overall control structure and the various components of the controller are shown in Figure $2 c$. This control structure will be referred to as the GLC-two degree of freedom output feedback structure.

\section{Closed-loop stability}

Input/Output Stability. The input/output stability of the overall closed-loop system is guaranteed as long as the parameters $\gamma_{\ell}, \ell=1, \ldots, r$ are chosen so that the roots of the characteristic equation:

$$
z^{r}+\gamma_{1} z^{r-1}+\ldots+\gamma_{r-1} z+\gamma_{r}=0
$$

lie inside the unit circle.

Internal Stability. In addition to the input/output stability, one must assure that the overall closed-loop system is internally stable: the state variables always remain bounded. If the overall 
closed-loop system is input/output-stable, and the conditions,

(i) the delay-free part of the process is hyperbolically minimum-phase

(ii) the roots of the characteristic equation

$$
z^{r}+\beta_{1} z^{r-1}+\ldots+\beta_{r-1} z+\beta_{r}=0
$$

lie inside the the unit circle, are satisfied, then the local internal stability of the closed-loop system under the controller of Theorem 3 will be guaranteed. For local internal stability of the closed-loop system under the controller of Theorem 2 , in addition to the conditions $\mathrm{i}$ and $\mathrm{i}$, the following condition,

(iii) the open-loop process is locally stable, must hold.

Remark 10. The tuning parameters $\beta_{\ell}$ 's in the controllers of Theorems 1, 2, and 3 do not affect the input/output dynamics of the overall closed-loop system; they characterize the hidden modes of the closed-loop dynamics. Apart from the requirement that the roots of $z^{r}+\beta_{1} z^{r-1}+\ldots+\beta_{r-1} z+\beta_{r}$ lie inside the unit circle, the choice of $\beta_{\ell}$ 's seems completely arbitrary. Note, however, that for robustness of the closed-loop dynamics, care must be taken so that the hidden modes are slower than the unmodeled dynamics.

\section{Error Feedback Control of Linear Systems}

Consider SISO discrete-time linear systems described by a state space model of the form:

$$
\left\{\begin{aligned}
x(k+1) & =A x(k)+B u(k) \\
y(k) & =C x(k)
\end{aligned}\right.
$$

where $A, B$, and $C$ are matrices of appropriate dimensions. This is a special case of Eq. 1 for:

$$
\begin{aligned}
\Phi[x(k), u(k)] & =A x(k)+B u(k) \\
h[x(k)] & =C x(k)
\end{aligned}
$$

Applying Definition 1 to the system of Eq. 43, we immediately see that the relative order $r$ of this system is the smallest integer for which

$$
C A^{r-1} B \neq 0
$$

and this is in complete agreement with the linear notion of relative order (Chen, 1984). Furthermore,

$$
\left\{\begin{aligned}
h^{\ell}(x) & =C A^{\ell} x, \quad \ell=0, \ldots, r-1 \\
h^{r-1}[\Phi(x, u)] & =C A^{r} x+C A^{r-1} B u
\end{aligned}\right.
$$

and the function $\Psi_{o}$, which was defined implicitly as the solution of Eq. 5 has the simple closed-form expression:

$$
\Psi_{o}(x, y)=\frac{1}{C A^{r-1} B}\left(y-C A^{r} x\right)
$$

The input/output behavior of the system of Eq. 43 can be represented by the $z$-domain transfer function:

$$
\frac{y(z)}{u(z)}=C(z I-A)^{-1} B
$$

Using the algebraic identity:

$$
z^{r} C(z I-A)^{-1} B=C A^{r}(z I-A)^{-1} B+C A^{r-1} B
$$

Equation 44 can be equivalently rewritten in the form:

$$
\frac{y(z)}{u(z)}=z^{-r}\left[C A^{r}(z I-A)^{-1} B+C A^{r-1} B\right]
$$

which provides a factorization of the transfer function of Eq. 44 into a pure delay, $z^{-r}$, and a delay-free part:

$$
H(z)=C A^{r}(z I-A)^{-1} B+C A^{r-1} B
$$

in terms of the system matrices $A, B$ and $C$.

The simplicity of the expressions for $\Phi, h^{l}$ and $\Psi_{o}$ in linear systems significantly simplifies the expressions for the derived control laws. In particular, the error-feedback controller of Corollary to Theorem 2 becomes:

$$
\left\{\begin{array}{r}
w(k+1)=\left(\begin{array}{r}
\left(C A^{r}-C\right)+\sum_{\ell=1}^{r-1} \gamma_{\ell}\left(C A^{r-\ell}-C\right) \\
C A^{r-1} B
\end{array}\right) w(k) \\
+B\left(\frac{1+\sum_{\ell=1}^{r} \gamma_{\ell}}{C A^{r-1} B}\right)_{e(k)} \\
u(k)=-\left(\frac{\left(C A^{r}-C\right)+\sum_{\ell=1}^{r-1} \gamma_{\ell}\left(C A^{r-\ell}-C\right)}{C A^{r-1} B}\right)_{w(k)}+\left(\frac{1+\sum_{\ell=1}^{r} \gamma_{\ell}}{C A^{r-1} B}\right)_{e(k)}
\end{array}\right.
$$

In particular, under the choice

$$
\gamma_{1}=-\alpha, \quad \gamma_{2}=\cdots=\gamma_{r}=0
$$

(which corresponds to requesting first-order-plus-dead-time response), the controller further simplifies into (see also Eq. 39):

$$
\left\{\begin{array}{r}
w(k+1)=\left(A-B \frac{C A^{r}-\alpha C A^{r-1}-(1-\alpha) C}{C A^{r-1} B}\right) w(k) \\
+B\left(\frac{1-\alpha}{C A^{r-1} B}\right) e(k) \\
u(k)=-\left(\frac{C A^{r}-\alpha C A^{r-1}-(1-\alpha) C}{C A^{r-1} B}\right) w(k) \\
+\left(\frac{1-\alpha}{C A^{r-1} B}\right) e(k)
\end{array}\right.
$$

It is straightforward to verify that Eq. 46 is a minimal statespace realization of the $z$-domain transfer function: 


$$
\frac{u(z)}{e(z}=\frac{1-\alpha}{1-\alpha z^{-1}-(1-\alpha) z^{-r}} \frac{1}{H(z)}
$$

where $H(z)$ is given by Eq. 45. The transfer function of Eq. 47 is exactly the controller derived from standard synthesis methods (for example, IMC) for a requested closed-loop response of the form:

$$
\frac{y(z)}{y_{s p}(z)}=z^{-r} \frac{1-\alpha}{1-\alpha z^{-1}}
$$

A similar development can be followed along the lines of the results of Theorem 3 for dead-time-free processes, resulting in a linear two-degree-of-freedom controller. Details are omitted for brevity.

\section{Model Algorithmic Control}

Model algorithmic control (MAC) is a one-step-ahead predictive controller, in which the control law is obtained from the minimization of the output error at time $k+r$. It uses an impulse response model to predict the future behavior of the process. MAC was developed in France in the late 70s within the chemical process industry. The original concept was established by Richalet et al. (1978), and later the theory was further advanced by Mehra et al. (1980) and Mehra and Rouhani (1980). This MPC technique has been implemented in many industrial processes such as PVC plant and distillation column (Richalet et al., 1978), steam generator (Lecrique et al., 1978), electric power plant (Mehra and Eterno, 1980), and flight control (Mehra et al., 1978).

In the work of Garcia and Morari (1982), a comprehensive review of MAC is provided and, more importantly, MAC is put into perspective and compared with theoretical controller synthesis methods. In particular, it is shown that the model algorithmic controller is essentially identical to the one arising from IMC.

The development of the MAC methodology is in terms of an input/output process model (in particular an impulse response model) although this is by no means a limitation. A state-space model could have been used following the conceptual steps of the methodology, leading to identical results. In what follows, we will develop a state-space reformulation of MAC for unconstrained linear processes whose delay-free part is minimum phase and then develop, for the first time, a nonlinear generalization of MAC. The nonlinear MAC will turn out to be identical to the GLC-error-feedback controller which was developed in the previous sections.

\section{State-space reformulation of MAC for unconstrained minimum-phase linear processes}

Consider linear processes described by a discrete-time state space model of the form of Eq. 43:

$$
\left\{\begin{aligned}
x_{m}(k+1) & =A x_{m}(k)+B u(k) \\
y_{m}(k) & =C x_{m}(k)
\end{aligned}\right.
$$

where the subscript $m$ has been added to explicitly indicate that $x_{m}$ and $y_{m}$ represent estimates of $x$ and $y$ obtained by simulating the model, given the manipulated input move $u(k)$. This notation will help differentiate $y_{m}$ from the measured output, which will still be denoted by $y$. The model of Eq. 48 can be simulated on-line to predict the future behavior of the process. In particular, from the model described by Eq. 48, we obtain:

$$
\begin{aligned}
y_{m}(k) & =C x_{m}(k) \\
y_{m}(k+1) & =C A x_{m}(k) \\
& \vdots \\
y_{m}(k+r-1) & =C A^{r-1} x_{m}(k) \\
y_{m}(k+r) & =C A^{r} x_{m}(k)+C A^{r-1} B u(k)
\end{aligned}
$$

from which one can predict the future changes in the output as follows:

$$
\begin{aligned}
y_{m}(k+1)-y_{m}(k) & =(C A-C) x_{m}(k) \\
y_{m}(k+2)-y_{m}(k) & =\left(C A^{2}-C\right) x_{m}(k) \\
& \vdots \\
y_{m}(k+r-1)-y_{m}(k) & =\left(C A^{r-1}-C\right) x_{m}(k) \\
y_{m}(k+r)-y_{m}(k) & =\left(C A^{r}-C\right) x_{m}(k)+C A^{r-1} B u(k)
\end{aligned}
$$

When these predicted changes are added to the measured output signal $y(k)$, one obtains future predictions of the output:

$$
\left\{\begin{aligned}
\hat{y}(k+1) & =y(k)+(C A-C) x_{m}(k) \\
\hat{y}(k+2) & =y(k)+\left(C A^{2}-C\right) x_{m}(k) \\
& \vdots \\
\hat{y}(k+r-1) & y(k)+\left(C A^{r-1}-C\right) x_{m}(k) \\
\hat{y}(k+r) & =y(k)+\left(C A^{r}-C\right) x_{m}(k)+C A^{r-1} B u(k)
\end{aligned}\right.
$$

where the superscript ${ }^{\wedge}$ is used to indicate that $\hat{y}$ represents a prediction of the output. It is interesting to observe that the output predictions in Eq. 49 are "closed-loop" predictions in the sense that they make use of the measured output signal. Also that the manipulated input move $u(k)$ affects the output after $r$ sampling periods, and this conforms with the interpretation of $r \Delta t$ as the overall delay of the system $[(r-1) \Delta t$ is the process dead time, and $\Delta t$ is the sampling delayl.

At every time step, the control computer can calculate the output predictions (Eq. 49), driven by $u(k)$ and $y(k)$, where $x_{m}(k)$ is obtained by on-line simulation of the state equations of Eq. 48 :

$$
x_{m}(k+1)=A x_{m}(k)+B u(k)
$$

The question that arises then is what should be the choice of $u(k)$ to obtain a desirable output response after $r$ time steps. If $u(k)$ is chosen so that $\hat{y}(k+r)$ is exactly the set-point value, this would clearly create a nonrobust situation. Instead, one can request $\hat{y}(k+r)$ to be in the right direction and cover a fraction of the "distance" between $\hat{y}(k+r-1)$ and the setpoint value. In other words, one can define a desirable value of the output at the $(k+r)$ th time step by:

$$
y_{d}(k+r)=(1-\alpha) y_{s p}+\alpha \hat{y}(k+r-1)
$$


where $\alpha$ is a tunable filter parameter such that $0<\alpha<1$. Clearly, $\alpha-0$ corresponds to $y_{d}(k+r) \rightarrow y_{s p}$ and, therefore, will try to force the output to go to set point as soon as possible, whereas $\alpha \rightarrow 1$ corresponds to $y_{d}(k+r)-\hat{y}(k+r-1)$, leaving the output unaffected. An intermediate choice of $\alpha$ corresponds to a desirable value of the output in between $y_{s p}$ and $\hat{y}(k+r-1)$ that tries to bridge the gap to a certain extent. Equation 50 is referred to as the "reference trajectory" in the MAC literature.

Once the reference trajectory has been specified, the question then becomes how to choose the control move $u(k)$ so that $\hat{y}(k+r)$ will match $y_{d}(k+r)$. This can be formulated as an optimization problem:

$$
\min _{u(k)}\left[y_{d}(k+r)-\hat{y}(k+r)\right]^{2}
$$

or, equivalently, in view of Eqs. 49 and 50:

$$
\begin{aligned}
\min _{u(k)}[(1-\alpha) e(k)- & {\left[\left(C A^{r}-C\right)\right.} \\
& \left.\left.-\alpha\left(C A^{r-1}-C\right)\right] x_{m}(k)-C A^{r+1} B u(k)\right]^{2}
\end{aligned}
$$

In the absence of the input constraints, this minimization problem is trivially solvable leading to the following control law:

$$
\begin{array}{r}
u(k)=-\left(\frac{C A^{r}-\alpha C A^{r-1}-(1-\alpha) C}{C A^{r-1} B}\right) \\
x_{m}(k) \\
+\left(\frac{1-\alpha}{C A^{r-1} B}\right) e(k)
\end{array}
$$

where $x_{m}(k)$ is obtained by simulating:

$$
x_{m}(k+1)=A x_{m}(k)+B u(k)
$$

This is identical to the controller realization of Eq. 46 with $x_{m}$ replacing $w$.

\section{Nonlinear MAC for unconstrained nonlinear processes}

The steps of the state-space linear MAC of the previous subsection can be extended "word by word" to nonlinear processes described by discrete-time models of the form of Eq. 1:

$$
\left\{\begin{array}{c}
x_{m}(k+1)=\Phi\left[x_{m}(k), u(k)\right] \\
y_{m}(k)=h\left[x_{m}(k)\right]
\end{array}\right.
$$

where again the subscript $m$ is added to indicate estimates of $x$ and $y$ obtained by model simulation and differentiate the simulated $y$ from the measured $y$.

On-line simulation of the model described by Eq. 52 can be used to predict the future changes in the output $y$ as follows:

$$
\begin{aligned}
y_{m}(k+1)-y_{m}(k) & =h^{1}\left[x_{m}(k)\right]-h\left[x_{m}(k)\right] \\
y_{m}(k+2)-y_{m}(k) & =h^{2}\left[x_{m}(k)\right]-h\left[x_{m}(k)\right] \\
& \vdots \\
y_{m}(k+r-1)-y_{m}(k) & =h^{r-1}\left[x_{m}(k)\right]-h\left[x_{m}(k)\right] \\
y_{m}(k+r)-y_{m}(k) & =h^{r-1}\left\{\Phi\left[x_{m}(k), u(k)\right]\right\}-h\left[x_{m}(k)\right]
\end{aligned}
$$

When these predicted changes are added to the measured output signal $y(k)$, one obtains the following "closed-loop" predictions of the output:

$$
\left\{\begin{aligned}
\hat{y}(k+1) & =y(k)+h^{1}\left[x_{m}(k)\right]-h\left[x_{m}(k)\right] \\
\hat{y}(k+2) & =y(k)+h^{2}\left[x_{m}(k)\right]-h\left[x_{m}(k)\right] \\
& \vdots \\
\hat{y}(k+r-1) & =y(k)+h^{r-1}\left[x_{m}(k)\right]-h\left[x_{m}(k)\right] \\
\hat{y}(k+r) & =y(k)+h^{r-1}\left\{\Phi\left[x_{m}(k), u(k)\right]\right\}-h\left[x_{m}(k)\right]
\end{aligned}\right.
$$

Defining a linear reference trajectory as same as in the linear case (Eq. 50):

$$
y_{d}(k+r)=(1-\alpha) y_{s p}+\alpha \hat{y}(k+r-1)
$$

one can derive a nonlinear MAC controller by requesting the output prediction to match the reference trajectory in the sense of minimizing the performance index of Eq. 51:

$$
\min _{u(k)}\left[y_{d}(k+r)-\hat{y}(k+r)\right]^{2}
$$

In view of Eqs. 50 and 53, this becomes:

$$
\begin{aligned}
\min _{u(k)}\left\{(1-\alpha) e(k)-h^{r-1}\{\Phi\right. & {\left.\left[x_{m}(k), u(k)\right]\right\} } \\
& \left.+\alpha h^{r-1}\left[x_{m}(k)\right]+(1-\alpha) h\left[x_{m}(k)\right]\right]^{2}
\end{aligned}
$$

In the absence of the input constraints, this minimization problem is trivially solvable. Minimizing $u(k)$ is the solution of the nonlinear algebraic equation:

$$
\begin{aligned}
h^{r-1}\left\{\Phi\left[x_{m}(k), u(k)\right]\right\}=\alpha h^{r-1}[ & \left.x_{m}(k)\right] \\
& +(1-\alpha) h\left[x_{m}(k)\right]+(1-\alpha) e(k)
\end{aligned}
$$

Remembering the definition of $\Psi_{o}$ (Eqs. 5 and 6), the solution can be represented as:

$$
\begin{aligned}
& u(k)=\Psi_{o}\left\{x_{m}(k), \alpha h^{r-1}\left[x_{m}(k)\right]\right. \\
&\left.+(1-\alpha) h\left[x_{m}(k)\right]+(1-\alpha) e(k)\right\}
\end{aligned}
$$

Thus, the derived control law is given by:

$$
\begin{aligned}
& \begin{array}{l}
u(k)=\Psi_{o}\left\{x_{m}(k), \alpha h^{r-1}\left[x_{m}(k)\right]+(1-\alpha) h\left[x_{m}(k)\right]\right. \\
+(1-\alpha) e(k)\}
\end{array} \\
& \text { where } x_{m}(k) \text { is obtained by simulating: } \\
& \qquad x_{m}(k+1)=\Phi\left[x_{m}(k), u(k)\right]
\end{aligned}
$$

which is identical to the controller realization of Eq. 39 with $x_{m}$ replacing $w$.

Remark 11. In the derivation of a MAC controller, it is conceivable to penalize the input by including an additional term in the performance index: 


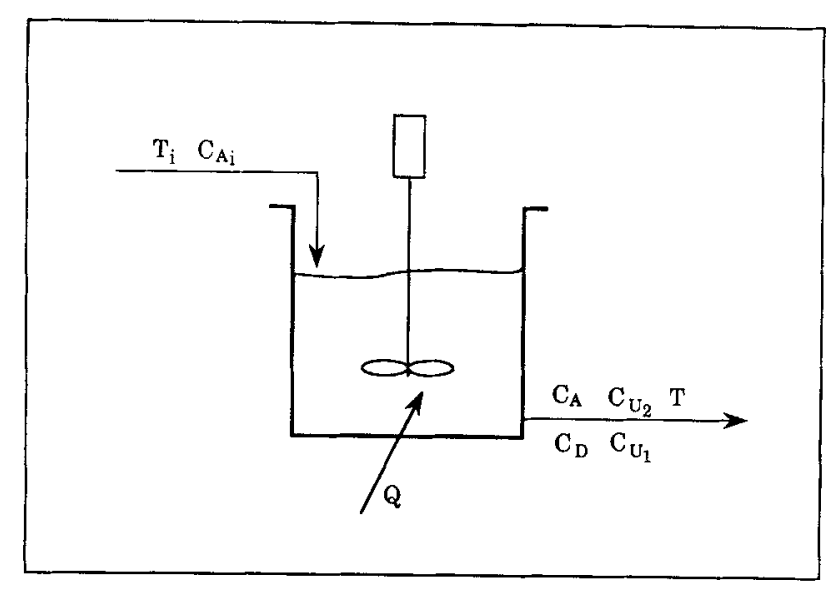

Figure 3. Simulated CSTR.

$$
\min _{u(k)}\left\{\left[y_{d}(k+r)-\hat{y}(k+r)\right]^{2}+\rho[u(k)]^{2}\right\}
$$

where $\rho$ is a positive tunable parameter (Mehra and Rouhani, 1980; see also Henson and Seborg, 1991b in a nonlinear MPC context). Minimization of such a performance index under no input constraints leads to a controller whose move $u(k)$ is the solution of the nonlinear algebraic equation:

$$
\begin{aligned}
& \frac{\rho u(k)}{\frac{\partial h^{r-1}\left\{\Phi\left[x_{m}(k), u(k)\right]\right\}}{\partial u(k)}}+h^{r-1}\left\{\Phi\left[x_{m}(k), u(k)\right]\right\} \\
& \quad=\alpha h^{r-1}\left[x_{m}(k)\right]+(1-\alpha) h\left[x_{m}(k)\right]+(1-\alpha) e(k)
\end{aligned}
$$

where $x_{m}(k)$ is obtained by simulating:

$$
x_{m}(k+1)=\Phi\left[x_{m}(k), u(k)\right]
$$

The above control law is not a GLC controller (does not induce a linear input/output behavior to the closed-loop system), and its theoretical properties are unknown at this point.

\section{Example: Application to a CSTR}

To study the performance of the proposed nonlinear control methodology, it is applied to a reactor system. The reactor is a CSTR (shown in Figure 3), in which the following parallel reactions

$$
\begin{aligned}
& A \stackrel{k_{1}}{\rightarrow} U_{1} \\
& A \stackrel{k_{2}}{\rightarrow} U_{2} \\
& A \stackrel{k_{d}}{\rightarrow} D
\end{aligned}
$$

take place. $U_{1}$ and $U_{2}$ are undesired side products, and $D$ is the desired product. The feed to the reactor does not contain $U_{1}, U_{2}$, or $D$. The dependence of the reaction rate constants $k_{1}, k_{2}$, and $k_{d}$ on temperature is given by $k_{i}=Z_{i} \exp$ $\left(-E_{a_{i}} / R T\right), i=1,2$ and $k_{d}=Z_{d} \exp \left(-E_{a_{d}} / R T\right)$.

\section{Mathematical model and control problem}

Energy and species mass balances for the reactor (under
Table 1. Parameters of the CSTR Model

\begin{tabular}{rlrl}
\hline$R$ & $=8.345 \times 10^{0}$ & & $\mathrm{~kJ} \cdot \mathrm{kmol}^{-1} \cdot \mathrm{K}^{-1}$ \\
$Z_{1}$ & $=2.00 \times 10^{3}$ & & $\mathrm{~m}^{6} \cdot \mathrm{kmol}^{-2} \cdot \mathrm{s}^{-1}$ \\
$Z_{2}$ & $=3.40 \times 10^{6}$ & & $\mathrm{kmol}{ }^{0.5} \cdot \mathrm{m}^{-1.5} \cdot \mathrm{s}^{-1}$ \\
$Z_{d}$ & $=2.63 \times 10^{5}$ & & $\mathrm{sJ} \cdot \mathrm{kmol}^{-1}$ \\
$E_{a_{1}}$ & $=4.90 \times 10^{4}$ & & $\mathrm{~kJ} \cdot \mathrm{kmol}^{-1}$ \\
$E_{a_{2}}$ & $=6.50 \times 10^{4}$ & $\mathrm{~kJ} \cdot \mathrm{kmol}^{-1}$ & $\mathrm{~kJ} \cdot \mathrm{kmol}^{-1}$ \\
$E_{a_{d}}$ & $=5.70 \times 10^{4}$ & $\mathrm{~kJ} \cdot \mathrm{kmol}^{-1}$ & $\mathrm{~kJ} \cdot \mathrm{kmol}^{-1}$ \\
$-\Delta H_{1}$ & $=4.50 \times 10^{4}$ & & \\
$-\Delta H_{2}$ & $=5.00 \times 10^{4}$ & & \\
$-\Delta H_{d}$ & $=6.00 \times 10^{4}$ & $\mathrm{~kg} \cdot \mathrm{m}^{-3}$ \\
$n_{1}$ & $=3.00 \times 10^{0}$ & & $\mathrm{~kJ} \cdot \mathrm{kg}^{-1} \cdot \mathrm{K}^{-1}$ \\
$n_{2}$ & $=5.00 \times 10^{-1}$ & &
\end{tabular}

standard assumptions) give the reactor model, which is of the form

$$
\left\{\begin{array}{l}
\frac{d C_{A}}{d t}=R_{A}+\frac{C_{A_{i}}-C_{A}}{\tau} \\
\frac{d T}{d t}=\frac{R_{H}}{\rho c}+\frac{T_{i}-T}{\tau}+\frac{Q}{\rho c V} \\
\frac{d C_{D}}{d t}=R_{D}-\frac{C_{D}}{\tau}
\end{array}\right.
$$

where the rate expressions $R_{A}, R_{H}$, and $R_{D}$ are given by:

$$
\begin{aligned}
& R_{A}=-k_{1} C_{A}^{n_{1}}-k_{2} C_{A}^{n_{2}}-k_{d} C_{A}^{n_{d}} \\
& R_{H}=\left(-\Delta H_{1}\right) k_{1} C_{A}^{n_{1}}+\left(-\Delta H_{2}\right) k_{2} C_{A}^{n_{2}}+\left(-\Delta H_{d}\right) k_{d} C_{A}^{n_{d}} \\
& R_{D}=k_{d} C_{A}^{n_{d}}
\end{aligned}
$$

The parameters of the reactor model are given in Table 1.

Figure $4 \mathrm{a}$ depicts the steady-state concentration of the desired product $\left(C_{D_{s s}}\right)$, given by:

$$
C_{D_{s s}}=\tau k_{d} C_{A_{s s}}^{n_{d}}
$$

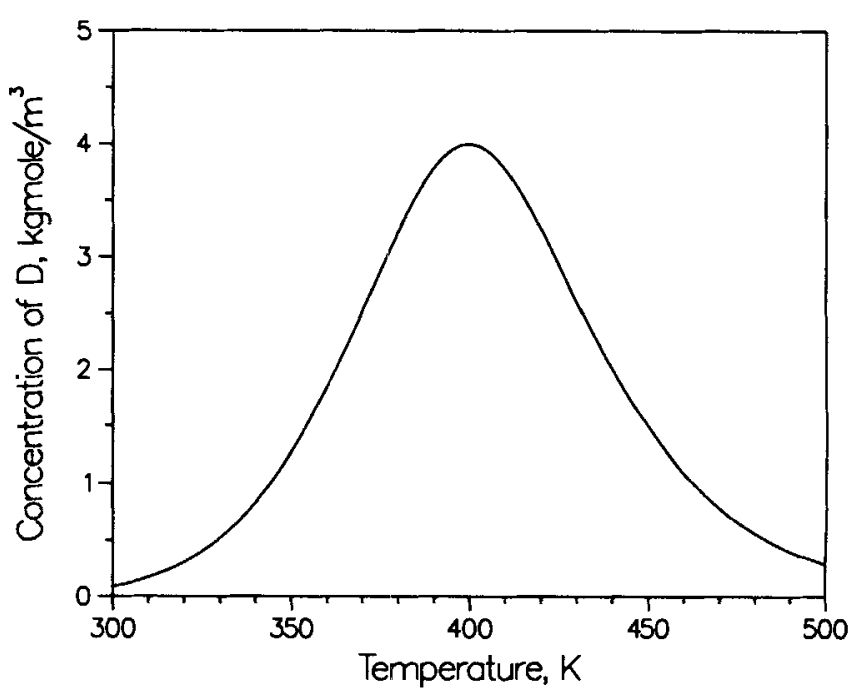

Figure 4a. Steady-state concentration of $D\left(C_{D_{s s}}\right)$ vs. steady-state temperature $\left(T_{s s}\right)$. 


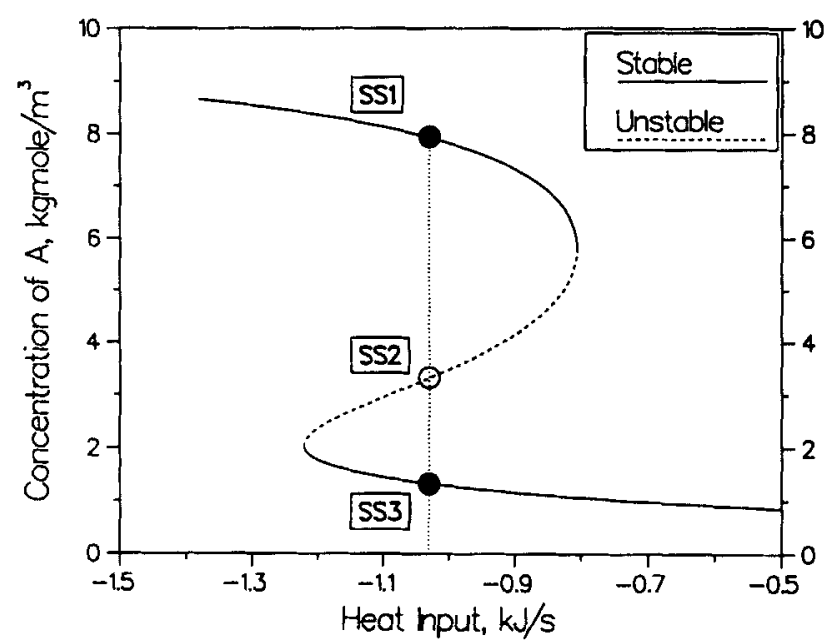

Figure 4b. Steady-state concentration of $A\left(C_{A_{s s}}\right)$ vs. steady-state heat input $\left(u_{s s}\right)$.

where $C_{A_{\mathrm{s}}}$ is the solution of:

$$
\tau\left(k_{1} C_{A_{s s}}^{n_{1}}+k_{2} C_{A_{s s}}^{n_{2}}+k_{d} C_{A_{s s}}^{n_{d}}\right)+C_{A_{s s}}=C_{A_{i}}
$$

vs. the steady-state temperature $T_{s s}$. As Figure 4 shows, the steady-state concentration of the desired product has a global maximum at $T_{s s}=400 \mathrm{~K}$, and this is independent of the inlet temperature $T_{i}$.

The control problem is to maintain the reactor temperature $T$ at $400 \mathrm{~K}$, which corresponds to maximum steady-state concentration of the desired product $\left(C_{D_{\mathrm{ss}}}=4.0 \mathrm{kmol} / \mathrm{m}^{3}\right)$, in the presence of process disturbances and modeling errors, by manipulating the heat input to the reactor $(Q)$. For the controller design, since $C_{D}$ does not affect $T$, only the first two differential equations of Eq. 54 are needed. Therefore, the process model, in standard state-space form, can be written as:

$$
\begin{aligned}
\int \frac{d}{d t}\left[\begin{array}{c}
C_{A} \\
T
\end{array}\right] & =\left[\begin{array}{l}
f_{1}\left(C_{A}, T\right) \\
f_{2}\left(C_{A}, T\right)
\end{array}\right]+\left[\begin{array}{c}
0 \\
\frac{1}{\rho c V}
\end{array}\right] u \\
y & =T
\end{aligned}
$$

Here, $x=\left[C_{A} T\right]^{T}, u=Q$, and

$$
\left[\begin{array}{l}
f_{1}\left(C_{A}, T\right) \\
f_{2}\left(C_{A}, T\right)
\end{array}\right]=\left[\begin{array}{c}
R_{A}\left(C_{A}, T\right)+\frac{C_{A_{i}}-C_{A}}{\tau} \\
\frac{R_{H}\left(C_{A}, T\right)}{\rho c}+\frac{T_{i}-T}{\tau}
\end{array}\right]
$$

An interesting feature of this control problem is the presence of steady-state multiplicities. Figures $4 \mathrm{~b}$ and $4 \mathrm{c}$ depict the steady-state concentration of $A\left(C_{A_{s}}\right)$ and the steady-state temperature $\left(T_{s s}\right)$ vs. steady-state heat input $\left(u_{s s}\right)$. As shown in these figures, for $u_{s s}=-1.030 \mathrm{~kJ} \cdot \mathrm{s}^{-1}$ there are three steadystate operating points (SS1, SS2, and SS3); it is at the highconversion, high-temperature, steady-state operating point (SS3) that the reactor must operate. The presence of steady-

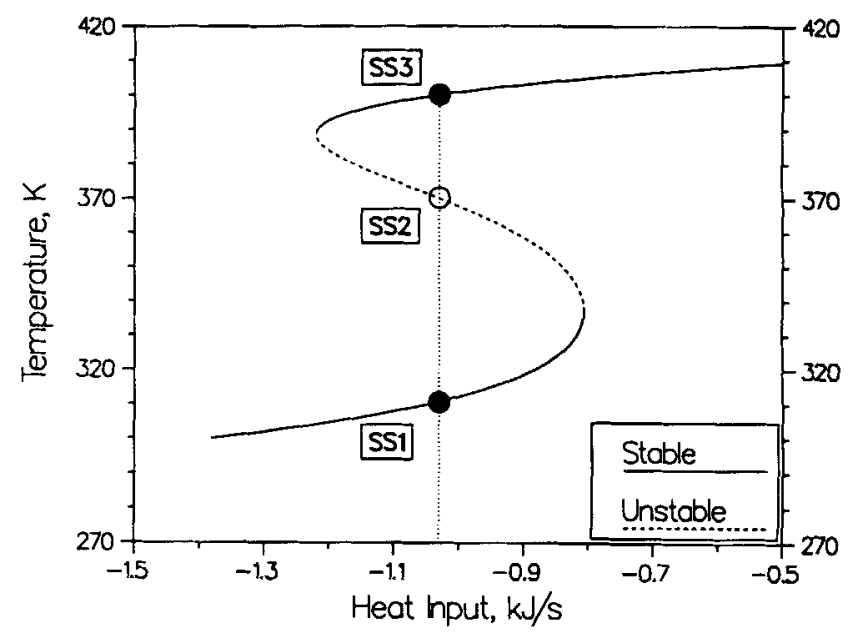

Figure 4c. Steady-state temperature $\left(T_{s s}\right)$ vs. steady-state heat input $\left(u_{s s}\right)$.

state multiplicities makes the reactor start-up a true control problem; if the necessary steady-state heat input $(-1.030$ $\left.\mathrm{kJ} \cdot \mathrm{s}^{-1}\right)$ is applied to the reactor in an open-loop fashion, the process will go to SS1 and not to SS3. Also the regulation problem is very important because process disturbances could potentially drive the reactor to the low-conversion, stable, steady-state operating point (SS1).

\section{Discrete-time model through forward difference ap- proximation}

For the discretization of the above continuous-time model, a simple forward difference method (Euler's method) is used, which can provide a good approximate discrete model under fast sampling. The resulting discrete-time model is:

$$
\left\{\begin{aligned}
{\left[\begin{array}{c}
C_{A}(k+1) \\
T(k+1)
\end{array}\right] } & =\left[\begin{array}{c}
C_{A}(k)+f_{1}\left(C_{A}(k), T(k)\right) \Delta t \\
T(k)+f_{2}\left(C_{A}(k), T(k)\right) \Delta t+\frac{\Delta t}{\rho c V} u(k)
\end{array}\right] \\
y(k) & =T(k)
\end{aligned}\right.
$$

where $\Delta t$ is the sampling period, which is in the form of Eq. 1. The nonlinear controllers will be synthesized on the basis of the discrete-time model of Eq. 55.

\section{Controller synthesis and implementation}

The first step is to calculate the relative order of the model of Eq. 55 according to Definition 1. Since

$$
\begin{gathered}
\frac{\partial h(x)}{\partial x}=\left[\begin{array}{ll}
0 & 1
\end{array}\right] \\
\frac{\partial \Phi(x, u)}{\partial u}=\left[\begin{array}{c}
0 \\
\frac{\Delta t}{\rho c V}
\end{array}\right]
\end{gathered}
$$


Table 2. Operating Conditions

\begin{tabular}{rlrl}
\hline$C_{A_{i}}$ & $=1.00 \times 10^{1}$ & & $\mathrm{kmol} \cdot \mathrm{m}^{-3}$ \\
$C_{A}(0)$ & $=1.00 \times 10^{-1}$ & & $\mathrm{kmol} \cdot \mathrm{m}^{-3}$ \\
$C_{D}(0)$ & $=0.00 \times 10^{0}$ & & $\mathrm{kmol} \cdot \mathrm{m}^{-3}$ \\
$T(0)$ & $=2.952 \times 10^{2}$ & & $\mathrm{~K}$ \\
$T_{s s}$ & $=4.00 \times 10^{2}$ & & $\mathrm{~kJ} \cdot \mathrm{s}^{-1}$ \\
$Q_{s s}$ & $=-1.0303 \times 10^{0}$ & & $\mathrm{kmol} \cdot \mathrm{m}^{-3}$ \\
$C_{A_{s s}}$ & $=1.3204 \times 10^{0}$ & & $\mathrm{~m}^{3}$ \\
$C_{D_{s s}}$ & $=4.000 \times 10^{0}$ & $\mathrm{~s}$ \\
$V$ & $=1.00 \times 10^{-2}$ & $\mathrm{~K}$ \\
$\tau$ & $=3.00 \times 10^{2}$ & & \\
\hline
\end{tabular}

and therefore

$$
\frac{\partial h(x)}{\partial x} \frac{\partial \Phi(x, u)}{\partial u}=\frac{\Delta t}{\rho c V} \neq 0
$$

the relative order is $r=1$.

The controllers of Theorems 1, 2 and 3, for this specific example, are as follows:

(A) "Basic" GLC: Full State Measurement. The feedback controller of Theorem 1 (Eq. 32) becomes:

$$
\left\{\begin{array}{c}
\xi(k+1)=\xi(k)+\left(1+\gamma_{1}\right) e(k) \\
u(k)=\left[\left(1+\beta_{1}\right)(\xi(k)-T(k))+\left(1+\gamma_{1}\right) e(k)\right. \\
\left.-f_{2}\left(C_{A}(k), T(k)\right) \Delta t\right] \frac{\rho c V}{\Delta t}
\end{array}\right.
$$

with the initial condition

$$
\xi(0)=T(0)
$$

(B) GLC-Error Feedback. The error-feedback controller of Theorem 2 (Eq. 36) takes the form:

$$
\left\{\begin{aligned}
& \xi(k+1)=\xi(k)+\left(1+\gamma_{1}\right) e(k) \\
& w_{1}(k+1)=w_{1}(k)+f_{1}\left(w_{1}(k), w_{2}(k)\right) \Delta t \\
& w_{2}(k+1)=w_{2}(k)+\left(1+\beta_{1}\right)\left[\xi(k)-w_{2}(k)\right]+\left(1+\gamma_{1}\right) e(k) \\
& u(k)=\left\{\left(1+\beta_{1}\right)\left[\xi(k)-w_{2}(k)\right]+\left(1+\gamma_{1}\right) e(k)\right. \\
&\left.-f_{2}\left[w_{1}(k), w_{2}(k)\right] \Delta t\right\} \frac{\rho c V}{\Delta t}
\end{aligned}\right.
$$

with the nominal initial conditions (see Table 2):

$$
\xi(0)=T(0), \quad w_{1}(0)=C_{A}(0), \quad w_{2}(0)=T(0)
$$

Since $\xi(0)=w_{2}(0)$, the above controller realization has one redundant state; $\xi(k)=w_{2}(k)$ for all $k$. Elimination of the redundant state leads to the reduced realization of the Corollary to Theorem 2 (Eq. 37):

$$
\left\{\begin{aligned}
w_{1}(k+1) & =w_{1}(k)+f_{1}\left[w_{1}(k), w_{2}(k)\right] \Delta t \\
w_{2}(k+1) & =w_{2}(k)+\left(1+\gamma_{1}\right) e(k) \\
u(k) & =\left[\left(1+\gamma_{1}\right) e(k)-f_{2}\left[w_{1}(k), w_{2}(k)\right] \Delta t\right] \frac{\rho c V}{\Delta t}
\end{aligned}\right.
$$

with the nominal initial conditions:

$$
w_{1}(0)=C_{A}(0), \quad w_{2}(0)=T(0)
$$

(C) GLC-Two Degree of Freedom Output Feedback. The two-degree-of-freedom controller of Theorem 3 (Eq. 42) takes the form (note that the system of Eq. 55 is already in normal form):

$$
\left\{\begin{aligned}
\xi(k+1) & =\xi(k)+\left(1+\gamma_{1}\right) e(k) \\
\eta(k+1) & =\eta(k)+f_{1}[\eta(k), T(k)] \Delta t \\
u(k) & =\left\{\left(1+\beta_{1}\right)[\xi(k)-T(k)]+\left(1+\gamma_{1}\right) e(k)\right. \\
& \left.-f_{2}[\eta(k), T(k)] \Delta t\right\} \frac{\rho c V}{\Delta t}
\end{aligned}\right.
$$

with the nominal initial conditions (see Table 2):

$$
\xi(0)=T(0), \quad \eta(0)=C_{A}(0)
$$

The above three controllers induce the same linear input/ output behavior:

$$
y(k+1)+\gamma_{1} y(k)=\left(1+\gamma_{1}\right) y_{s p}(k)
$$

to the overall closed-loop system.

Some comments must be made here with regard to the nature of the derived controllers:

- All three controllers possess integral action: the state $\xi$ represents the integral of the error multiplied by $\left(1+\gamma_{1}\right) / \Delta t$.

- The origin of the integral of the error could be seen from the nature of the requested closed-loop response (Eq. 60). Rearranging terms in Eq. 60, we see that all three controllers are trying to enforce the error-to-output relationship

$$
y(k+1)-y(k)=(1+\gamma) e(k)
$$

that is, they are trying to match the system output with the integral of the error multiplied by $\left(1+\gamma_{1}\right) / \Delta t$. Each controller uses a different strategy to enforce this matching, but in all three cases, the integral of the error arises in the control law.

- In the derivation of the realization of Eq. 57, an openloop observer was used: $w_{1}$ and $w_{2}$ represent the estimates of the concentration $C_{A}$ and temperature $T$, respectively, via openloop simulation of the process model. Once the input/output linearizing state feedback has been substituted under $\xi(0)=w_{2}$ $(0), w_{2}$ becomes the integral of the error multiplied by $\left(1+\gamma_{1}\right) /$ $\Delta t$, and this gives rise to the reduction of the order of the realization (Eq. 58). This observation indicates that although the output feedback controller was derived via brute force state feedback/state observer combination, the interpretation of the controller states is much more subtle.

- Controller A uses an on-line measurement of the concentration of $A$ whereas controllers B and C both simulate the $A$-balance equation on-line in order to obtain an estimate of $C_{A}$. In particular, the $\eta$-equation of controller $\mathrm{C}$ is exactly the $A$-balance equation and is driven by the on-line measurement of the reactor temperature. In controller B (Eq. 58), the $w_{1}$ equation is exactly the $A$-balance equation and is driven by $w_{2}$ 
Table 3. List of the Simulation Cases

\begin{tabular}{cccc}
\hline GLC Structure & $\begin{array}{c}\text { Nominal } \\
\text { Model }\end{array}$ & $\begin{array}{c}\text { Modeling } \\
\text { Error }\end{array}$ & $\begin{array}{c}\text { Initialization } \\
\text { Error }\end{array}$ \\
\hline "Basic" & A1 & A2 & - \\
Error-Feedback & B1 & B2 & B3 \\
Two-Degree-of-Freedom & C1 & C2 & C3
\end{tabular}

[integral of the error multiplied by $\left(1+\gamma_{1}\right) / \Delta t$ ] rather than the on-line measurement of the reactor temperature. Although $w_{2}$ is supposed to match the reactor temperature in closed-loop, disturbances and modeling errors will create a mismatch, which will impact upon the quality of the concentration estimate.

\section{Simulation Results}

Using the operating conditions given in Table 2, numerical simulations are performed to examine the servo and regulatory performance of the three controllers A, B and C. In particular, the objective is to investigate the ability of the three controllers in:

- Performing a smooth and fast reactor start-up

- Rejecting the effect of a step change ( $20 \mathrm{~K}$ increase) in the inlet temperature $T_{i}$.

To simulate the reactor process, the standard software package ODEPACK is used to integrate numerically the ordinary differential equations in Eq. 54. Every $2.5 \mathrm{~s}$ (the sampling period $\Delta t$ ), the value(s) of $C_{A}$ and/or $T$ calculated by the ODE solver are used in the discrete-time controllers as on-line process measurements.

In what follows, the servo and regulatory performance of the three controllers $\mathrm{A}, \mathrm{B}$ and $\mathrm{C}$ are investigated under: (1) perfect model (nominal case); (2) modeling errors (20\% error in the frequency factors of all the reactions); and (3) observer initialization errors $\left[w_{1}(0)=C_{A}(0)+1\right.$ for Case $B 3$, and $\eta(0)=C_{A}(0)+1$ for Case C3]. A list of the simulation cases is provided in Table 3 . The tunable parameters of the controllers are chosen to be $\beta_{1}=\gamma_{1}=-0.96$.

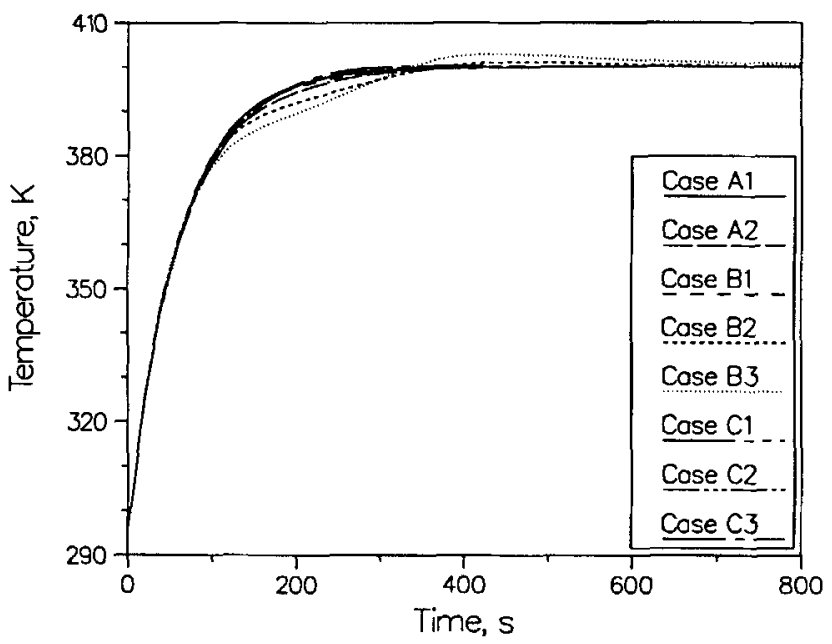

Figure 5a. Profiles of the output during start-up period.

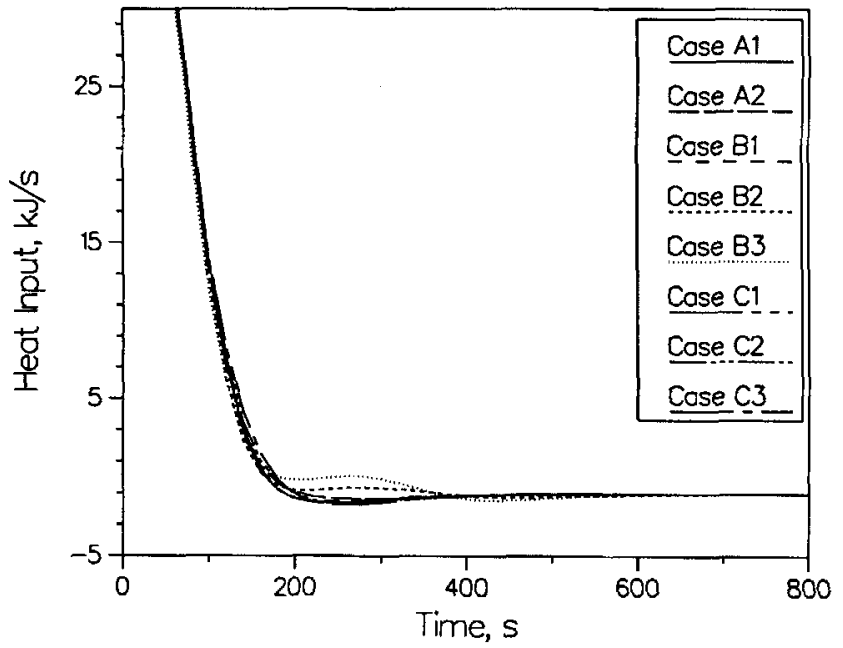

Figure 5b. Profiles of the manipulated input corresponding to Figure $5 a$.

\section{Start-Up Performance}

Figure 5a depicts the start-up profiles of the output under the three controllers and different conditions. The output profiles in Cases A1, A2, B1, C1, C2 and C3 are almost identical and follow the requested first-order closed-loop response. The slight loss in performance in Case B2 (controller B with the modeling errors) and in Case B3 (controller B with the observer initialization errors) is insignificant relative to the magnitude of these errors. Overall, Figure 5a demonstrates the robustness of the controllers to the modeling and observer initialization errors. Figure $5 \mathrm{~b}$ shows the corresponding manipulated input profiles for the start-up period.

Figures $5 \mathrm{c}$ and $5 \mathrm{~d}$ compare the variations of actual and estimated values of the state variable $C_{A}$ during the period of start-up for Cases B2, B3, C2 and C3. (In Cases B1 and C1, which are not shown, $C_{A}$ is perfectly estimated.) As expected, in the absence of modeling errors (Cases B3 and C3), the

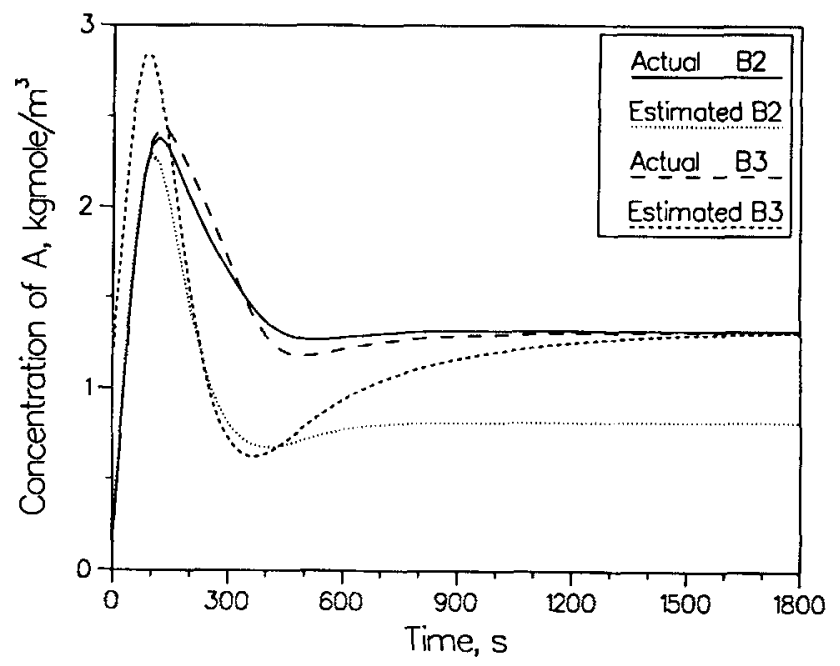

Figure 5c. Actual and estimated values of concentration of $\boldsymbol{A}$ corresponding to Figure 5 a (Controller B). 


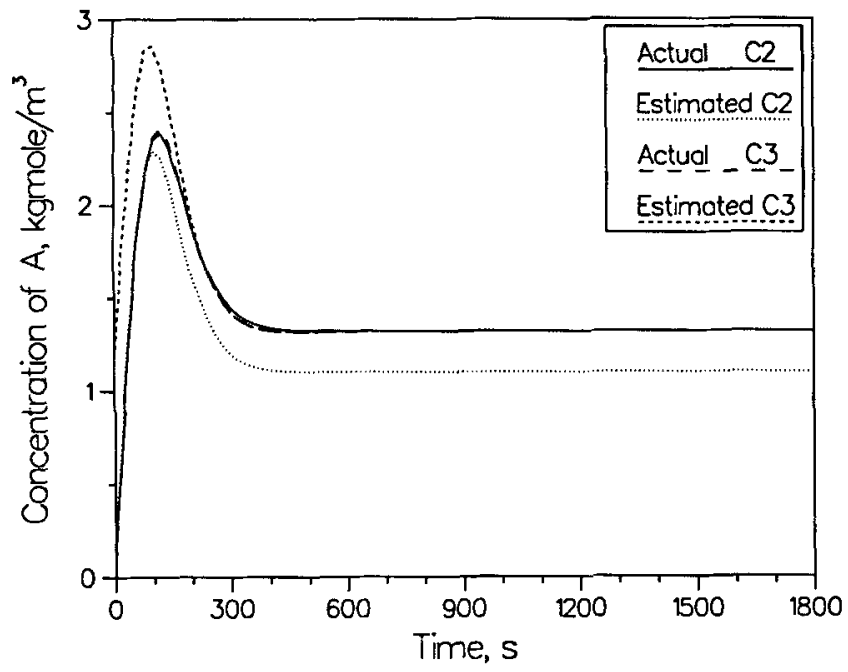

Figure 5d. Actual and estimated values of concentration of $A$ corresponding to Figure 5 a (Controller C).

estimation error goes asymptotically to zero. However, in Cases $\mathrm{B} 2$ and $\mathrm{C} 2$ (controllers $\mathrm{B}$ and $\mathrm{C}$ with modeling errors), there is a permanent mismatch between the actual and estimated values of $C_{A}$. This does not affect controller performance; the offset of the state estimate is rejected in the overall control structure.

\section{Regulatory Performance}

Figure 6a (which is a continuation of Figure 5a) depicts the regulatory performance of the controllers in rejecting the effect of an unmeasurable step disturbance in $T_{i}$ (20 degrees increase, from $22^{\circ} \mathrm{C}$ to $42^{\circ} \mathrm{C}$, at $t=2,000 \mathrm{~s}$ ). The output profiles in Cases $\mathrm{A} 1, \mathrm{~A} 2, \mathrm{C} 1$ and $\mathrm{C} 2$ are almost identical and correspond to almost perfect rejection of the disturbance. In Cases B1 and $\mathrm{B} 2$, the rejection of the disturbance is significantly slower, but still satisfactory relative to the magnitude of the applied dis-

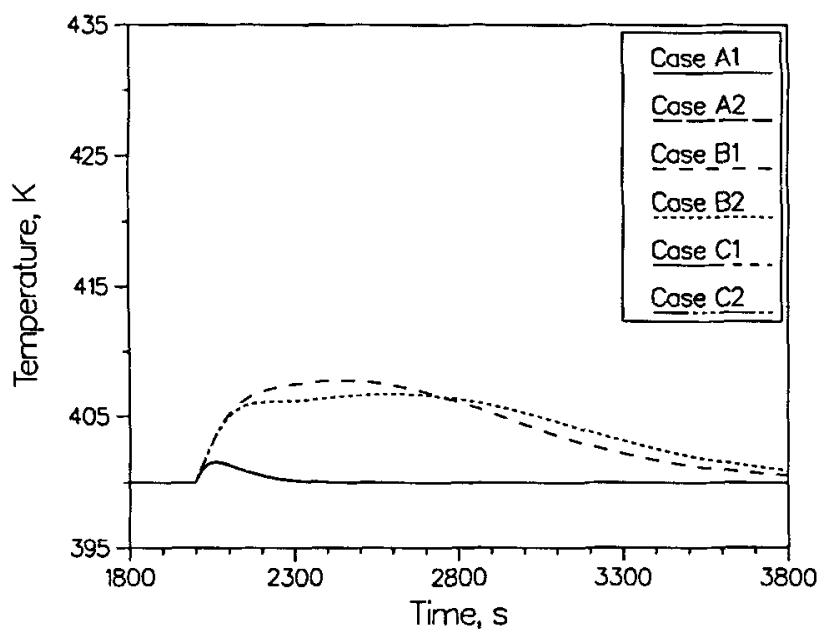

Figure 6a. Profiles of the output when there is a step change in $T_{i}$ at $t=2,000 \mathrm{~s}$.

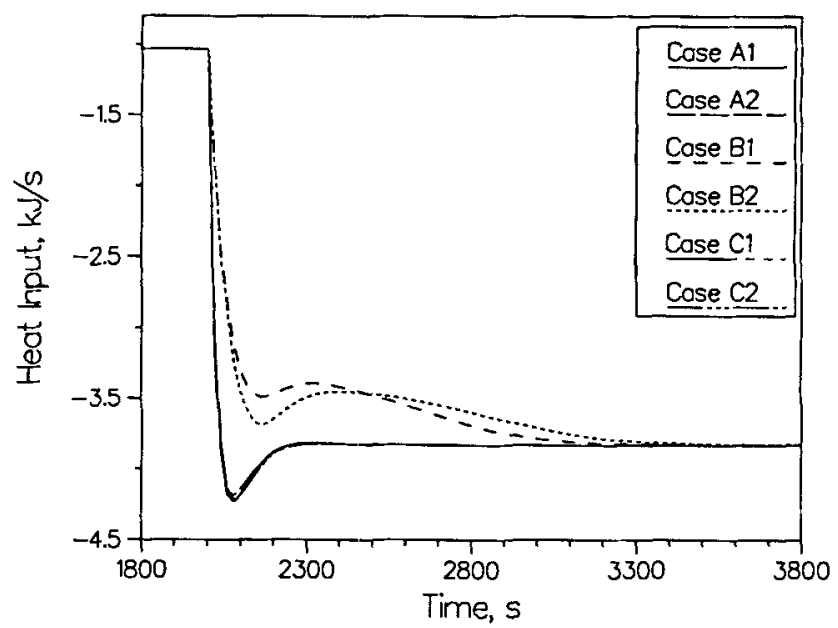

Figure 6b. Profiles of the manipulated input corresponding to Figure $6 a$.

turbance. Figure $6 \mathrm{~b}$ (which is a continuation of Figure $5 \mathrm{~b}$ ) shows the corresponding manipulated input profiles. The manipulated input profiles for Cases B1 and B2 are less aggressive compared to the other cases. It should be noted that the output and manipulated input profiles for Cases B3 and C3 (which are not shown here) are identical to ones for Cases $\mathrm{B} 1$ and $\mathrm{C1}$, respectively, since the initialization errors have already been rejected.

Figures $6 \mathrm{c}$ and $6 \mathrm{~d}$ (which are continuations of Figures 5c and $5 \mathrm{~d}$, respectively) compare the variations of the actual and estimated values of the state variable $C_{A}$ for the period 1,800 $\leq t<3,800 \mathrm{~s}$. In Cases $\mathrm{B} 2$ and $\mathrm{B} 3$, the step change in $T_{i}$ creates a huge difference between actual and estimated values of $C_{A}$ for $t>2,000 \mathrm{~s}$. This estimation error is responsible for the sluggish regulatory behavior shown in Figure 6a. Despite the large estimation error, controller B is capable of rejecting the disturbance eventually. On the other hand, in Cases C2 and C3, the step change in $T_{i}$ at $t=2,000 \mathrm{~s}$ does not deteriorate the observer performance, because the reduced-order observer

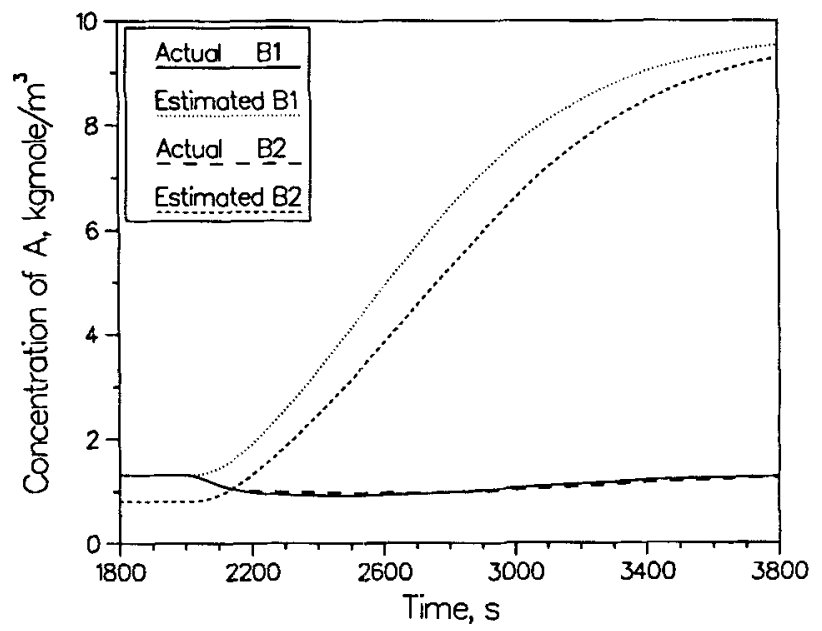

Figure 6c. Actual and estimated values of concentration of $A$ corresponding to Figure 6 a (Controller B). 


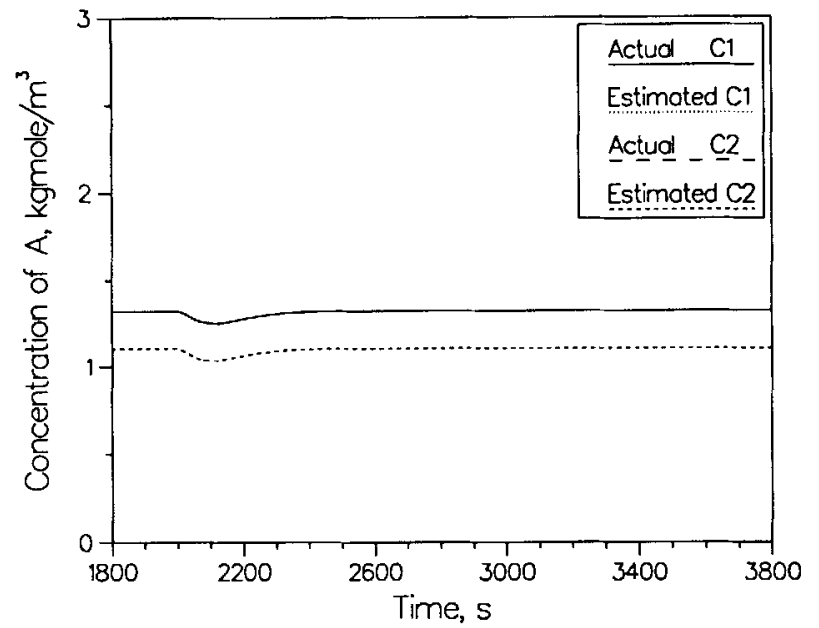

Figure 6d. Actual and estimated values of concentration of $A$ corresponding to Figure 6 a (Controller C).

uses the on-line measurement of the reactor temperature to estimate $C_{A}$. Despite the discrepancy between actual and estimated $C_{A}$ in Case $\mathrm{C} 2$, the controller shows the same performance as in Cases $\mathrm{C} 1$ and $\mathrm{C} 3$. Overall, controller $\mathrm{C}$ (with reduced-observer) is more effective than controller $\mathrm{B}$ (with fullorder observer) in rejecting the particular disturbance.

The above simulation results show the satisfactory performance of the three controllers under the large observer initialization and modeling errors. They also confirm that the best controller performance can be achieved when all the states are measured on-line. In this specific example, the adverse effect of the unmeasurable disturbance on the observer performance is more significant than the effects of modeling and observer initialization errors.

\section{Acknowledgment}

Financial support from the National Science Foundation through the grant CTS-8912836 is gratefully acknowledged.

\section{Notation}

\footnotetext{
$A=$ reactant

$c=$ heat capacity of reacting mixture, $\mathrm{kJ} \cdot \mathrm{kg}^{-1} \cdot \mathrm{K}^{-1}$

$C_{A}=$ concentration of reactant $A, \mathrm{kmol} \cdot \mathrm{m}^{-3}$

$C_{A_{s s}}=$ steady state concentration of reactant $A, \mathrm{kmol} \cdot \mathrm{m}^{-3}$

$C_{A_{i}}=$ inlet concentration of reactant, $A, \mathrm{kmol} \cdot \mathrm{m}^{-3}$

$C_{D}=$ concentration of desired product, $\mathrm{kmol} \cdot \mathrm{m}^{-3}$

$D_{D_{s s}}=$ steady state concentration of desired product, $\mathrm{kmol} \cdot \mathrm{m}^{-3}$

$C_{U_{t}}=$ concentration of undesired product $U_{p}, \mathrm{kmol} \cdot \mathrm{m}^{-3}$

$D=$ desired product

$E_{a_{d}}=$ activation energy for desirable reaction, $\mathrm{kJ} \cdot \mathrm{kmol}^{-1}$

$E a_{\ell}=$ activation energy for undesirable reaction $\ell, \mathrm{kJ} \cdot \mathrm{kmol}^{-1}$

$n_{d}=$ order of the desirable reaction

$n_{\ell}=$ order of the undesirable reaction $\ell$

$Q=$ rate of heat input to reactor, $\mathrm{kJ} \cdot \mathrm{s}^{-1}$

$Q_{s s}=$ steady-state rate of heat input to reactor, $\mathrm{kJ} \cdot \mathrm{s}^{-1}$

$r=$ relative order of controlled output $y$

$R=$ universal gas constant, $\mathrm{kJ} \cdot \mathrm{kmol}^{-1} \cdot \mathrm{K}^{-1}$

$R_{A}, R_{D}=$ rate of production of $A$ and $D$, respectively, $\mathrm{kmol} \cdot \mathrm{m}^{-3} \cdot \mathrm{s}^{-1}$
}

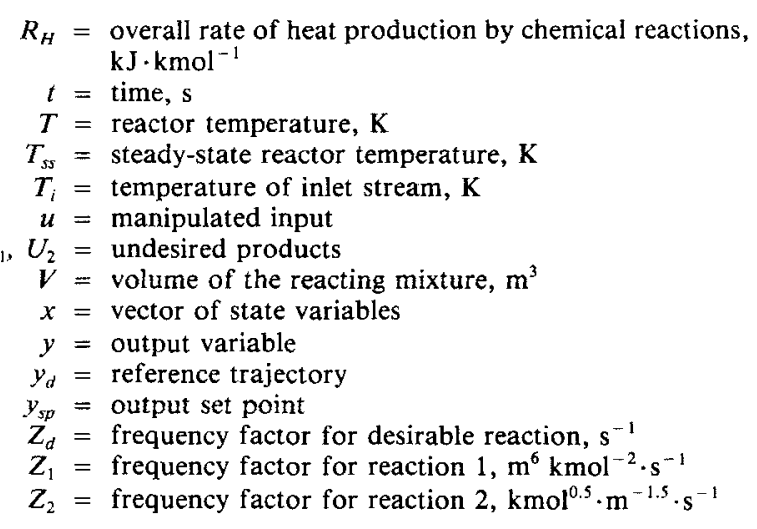

\section{Greek letters}

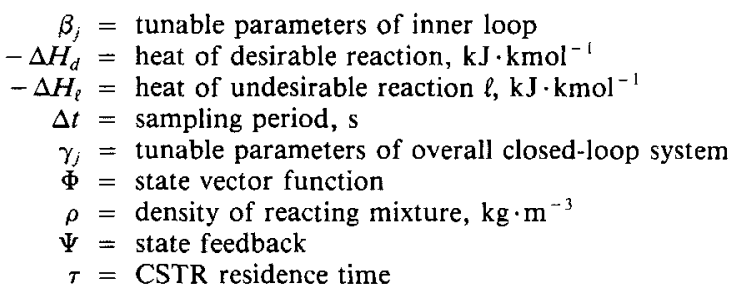

Math symbols

$$
\begin{aligned}
\triangleq & =\text { is defined } \\
\epsilon & =\text { belong to } \\
\mathbb{R} & =\text { real line } \\
0 & =\text { composition of functions }
\end{aligned}
$$

\section{Acronyms}

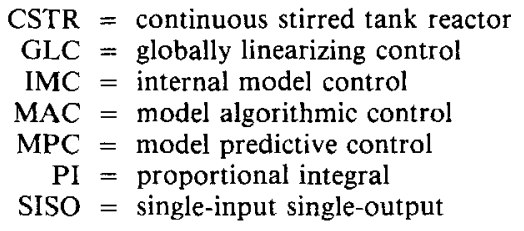

\section{Literature Cited}

Alevisakis, G., and D. E. Seborg, "An Extension of the Smith Predictor Method to Multivariable Linear Systems Containing Time Delays," Ind. J. Control, 3, 541 (1973).

Bequette, B. W., "Nonlinear Control of Chemical Processes: A Review," Ind. Eng. Chem. Res., 30, 1391 (1991).

Biegler, L. T, and J. B. Rawlings, "Optimization Approaches to Nonlinear Model Predictive Control," CPC IV, 543 (1991).

Chen, C. T., Linear System Theory and Design, Holt, Rinehart and Winston, 301 (1984).

Cutler, C. R., and B. L. Ramaker, "Dynamic Matrix Control-A Computer Control Algorithm," AIChE Meeting, Houston (1979).

Daoutidis, P., and C. Kravaris, "Dynamic Output Feedback Control of Minimum-Phase Nonlinear Processes," Chem. Eng. Sci., 47, 837 (1992).

Daoutidis, P., M. Soroush, and C. Kravaris, "Dynamic Output Feedback Control of Multivariable Nonlinear Processes," Paper No. 149b, AIChE Meeting, Los Angeles (1991).

Economou, C. G., M. Morari, and B. O. Palsson, "Internal Model Control: 5. Extension to Nonlinear Systems," Ind. Eng. Process Des. Dev., 25, 403 (1986).

Garcia, C. E., and M. Morari, "Internal Model Control: 1. A Unifying 
Review and Some New Results," Ind. Eng. Chem. Process Des. Dev., 21, 308 (1982).

Glad, S. T., "Output Dead-Beat Control for Nonlinear Systems with One Zero at Infinity," Sys. \& Cont. Lett., 9, 249 (1987).

Grizzle, J. W., "Local Input/Output Decoupling of Discrete-Time Nonlinear Systems," Int. J. Control, 43, 1517 (1986).

Henson, M. A., and D. E. Seborg, "An Internal Model Control Strategy for Nonlinear Systems," AIChE J., 37, 1065 (1991a).

Henson, M. A., and D. E. Seborg, "Theoretical Analysis of Unconstrained Nonlinear Model Predictive Control,' Paper No. 145a, AIChE Meeting, Los Angeles (1991b).

Hernandez, E., and Y. Arkun, "Modeling and Control of Nonlinear Systems Using Polynomial ARMA Models," Paper No. 149e, AIChE Meeting, Los Angeles (1991).

Hidalgo, P. M., and C. B. Brosilow, "Nonlinear Model Predictive Control of Styrene Polymerization at Unstable Operating Points," Comp. Chem. Eng., 14, 481 (1990).

Isermann, R., Digital Control Systems, 2nd ed., Vol. 1, SpringerVerlag, 68 (1989).

Isidori, A., Nonlinear Control Systems: An Introduction, 2nd ed., Springer-Verlag, 156 (1989).

Li, W. C., L. T. Biegler, C. G. Economou, and M. Morari, "A Constrained Pseudo-Newton Control Strategy for Nonlinear Systems," Comp. Chem. Eng., 14, 451 (1990).

Kravaris, C., "Input/Output Linearization: A Nonlinear Analog of Placing Poles at the Process Zeros," AIChE J., 34, 11, 1803 (1988).

Kravaris, C., and C. B. Chung, "Nonlinear State Feedback Synthesis by Global Input/Output Linearization," AIChE J., 33, 592 (1987).

Kravaris, C., and J. C. Kantor, "Geometric Methods for Nonlinear Process Control: 1. Background," Ind. Eng.Chem. Res., 29, 2295 (1990a).

Kravaris, C., and J. C. Kantor, "Geometric Methods for Nonlinear Process Control: 2. Controller Synthesis," Ind. Eng. Chem. Res., 29, 2311 (1990b).

Kravaris, C., and M. Soroush, "Synthesis of Multivariable Nonlinear Controllers by Input/Output Linearization," AIChE J., 36, 249 (1990).

Kravaris, C., and Y. Arkun, "Geometric Nonlinear Control-An Overview," CPC IV, 479 (1991).

Lecrique, M., A. Rault, M. Tessier, and J. L. Testud, "Multivariable Regulation of a Thermal Power Plant Steam Generator," IFAC World Cong., Helsinki (1978).

McLellan, P. J., T. J. Harris, and D. W. Bacon, "Error Trajectory Descriptions of Nonlinear Controller Designs," Chem. Eng. Sci., 45, 3017 (1990).

Mehra, R. K., and R. Rouhani, "Theoretical Considerations on Model Algorithmic Control for Nonminimum Phase Systems,"' Proc. ACC, TA8-B (1980).

Mehra, R. K., and J. S. Eterno, "Model Algorithmic Control for Electric Power Plants," Proc, IEEE CDC, Albuquerque, NM (1980).

Mehra, R. K., R. Rouhani, and R. Praly, "New Theoretical Developments in Multivariable Predictive Algorithmic Control," Proc. ACC, FA9-B (1980).

Mehra, R. K., W. C. Kessel, A. Rault, J. Richalet, and J. Papon, "Model Algorithm Control Using IDCOM for the F100 Jet Engine Multivariable Control Design Problem," Alternatives for Linear Multivariable Control, Sain, Peczkowski and Melsa, eds., NEC, Inc., Chicago, 317 (1978).

Monaco, S., and D. Normand-Cyrot, "Zero Dynamics of Sampled Nonlinear Systems," Sys. \& Cont. Lett., 11, 229 (1988).

Monaco, S., D. Normand-Cyrot, and T. Isola, "Nonlinear Decoupling in Discrete Time," IFAC Nonlinear Control Systems Design, Capri, Italy, 99 (1989).

Nijmeijer, H., and A. J. van der Schaft, Nonlinear Dynamical Control Systems, Springer-Verlag, 437 (1990).

O'Reilly, J., "The Discrete Linear Time Invariant Time-Optimal Control Problem-An Overview," Automatica, 17, 363 (1981).

Pathwardhan, A. A., J. B. Rawlings, and T. F. Edgar, "Nonlinear Model Predictive Control," Chem. Eng. Comm., 87, 123 (1990).

Prett, D. M., and R. D. Gillette, "Optimization and Constrained Multivariable Control of a Catalytic Cracking Unit," AIChE Meeting, Houston (1979).

Richalet, J., A. Rault, J. L. Testud, and J. Papon, "Model Predictive
Heuristic Control: Application to Industrial Processes," Automatica, 14, 413 (1978).

Rouhani, R., and R. K. Mehra, "Model Algorithmic Control (MAC): Basic Theoretical Properties," Automatica, 18, 401 (1982).

Sistu, P. B., and B. W. Bequette, "Nonlinear Predictive Control of Uncertain Chemical Processes: Application to a CSTR," AIChE J., 37, 1711 (1991).

Soroush, M., and C. Kravaris, "Nonlinear Control of a Batch Polymerization Reactor: An Experimental Study," AIChE J., 38, 1429 (1992a).

Soroush, M., and C. Kravaris, "Multivariable Nonlinear Control of a Continuous Polymerization Reactor," Proc. ACC, 607 (1992b).

Stubbs, D., and S. A. Svoronos, "A Simple Controller via Feedback Linearization and its Use in Adaptive Control," Proc. ACC, 1857 (1989).

\section{Appendix: Proofs}

Proof of Proposition 1. Since the delay-free part of the system of Eq. 1 is hyperbolically minimum phase, this means (by Definition 3) that all the eigenvalues of the matrix $S_{o}(0,0)$, where $J_{o}$ is given by Eq. 11 , are in the interior of the unit circle. Using the definition of relative order and standard matrix identities, one finds the characteristic polynomial of the matrix $\mathfrak{I}_{o}(0,0)$ to be:

$$
\frac{z \frac{\partial h(0)}{\partial x} \operatorname{adj}\left[z I-\frac{\partial \Phi(0,0)}{\partial x}\right] \frac{\partial \Phi(0,0)}{\partial u}}{\frac{\partial h(0)}{\partial x}\left[\frac{\partial \Phi(0,0)}{\partial x}\right]^{r-1} \frac{\partial \Phi(0,0)}{\partial u}}
$$

Therefore, the roots of the polynomial:

$$
\frac{\partial h(0)}{\partial x} \operatorname{adj}\left(z l-\frac{\partial \Phi(0,0)}{\partial x}\right) \frac{\partial \Phi(0,0)}{\partial u}
$$

all lie in the interior of the unit circle.

On the other hand, the local asymptotic stability characteristics of the closed-loop system of Eq. 25 are determined by the eigenvalues of the matrix $\mathfrak{I}_{\beta}(0,0)$, where $\mathfrak{S}_{\beta}$ is given by Eq. 26. Using the definition of relative order and standard matrix identities, one finds the characteristic polynomial of the matrix $\mathfrak{J}_{\beta}(0,0)$ to be:

$$
\frac{\left(z^{r}+\beta_{1} z^{r-1}+\cdots+\beta_{r-1} z+\beta_{r}\right) \frac{\partial h(0)}{\partial x} \text { adj }\left[z I-\frac{\partial \Phi(0,0)}{\partial x}\right] \frac{\partial \Phi(0,0)}{\partial u}}{\frac{\partial h(0)}{\partial x}\left[\frac{\partial \Phi(0,0)}{\partial x}\right]^{r-1} \frac{\partial \Phi(0,0)}{\partial u}}
$$

Because the roots of

$$
z^{r}+\beta_{1} z^{r-1}+\cdots+\beta_{r-1} z+\beta_{r}=0
$$

are assumed to be all in the interior of the unit circle, it follows that all the eigenvalues of the matrix $\mathfrak{I}_{\beta}(0,0)$ will be in the interior of the unit circle and therefore the closed-loop system of Eq. 25 will be locally asymptotically stable.

Proof of Theorem 1. Define the auxiliary variable:

$$
v(k)=\frac{C_{c} \xi(k)+D_{c}\left[y_{s p}(k)-y(k)\right]}{\beta_{0}}
$$


Then, Eq. 32 can be viewed as being composed of two subsystems:

$$
\left\{\begin{aligned}
\xi(k+1) & =A_{c} \xi(k)+B_{c}\left[y_{s p}(k)-y(k)\right] \\
v(k) & =\frac{C_{c} \xi(k)+D_{c}\left[y_{s p}(k)-y(k)\right]}{\beta_{0}}
\end{aligned}\right.
$$

and

$$
u(k)=\Psi_{o}\left\{x(k), \beta_{0} v(k)-\sum_{\ell=1}^{r} \beta_{\ell} h^{r-\ell}[x(k)]\right\}
$$

The subsystem of Eq. A1 is the realization of the external controller of Eq. 31, whose input/output behavior can be described by the difference operator representation:

$$
\begin{aligned}
& \frac{\left(q^{r}-1\right)+\gamma_{1}\left(q^{r-1}-1\right)+\cdots+\gamma_{r-1}(q-1)}{1+\gamma_{1}+\cdots+\gamma_{r-1}+\gamma_{r}} v(k) \\
& =\frac{1}{\beta_{0}}\left(q^{r}+\beta_{1} q^{r-1}+\cdots+\beta_{r}\right)\left[y_{s p}(k)-y(k)\right]
\end{aligned}
$$

where $q$ is the forward shift operator. On the other hand, the subsystem of Eq. A2 is the input/output linearizing feedback law of Eq. 22, which induces the closed-loop response:

$$
y(k+r)+\sum_{\ell=1}^{r} \beta_{\ell} y\left(k+r-\mathfrak{l}=\beta_{0} v(k)\right.
$$

or, equivalently,

$$
\frac{1}{\beta_{0}}\left(q^{r}+\beta_{1} q^{r-1}+\cdots+\beta_{r-1} q+\beta_{r}\right) y(k)=v(k)
$$

Combining Eqs. A3 and A4, we obtain the desired closed-loop input/output dynamics:

$$
\begin{aligned}
\left(q^{r}+\gamma_{1} q^{-1}+\cdots+\gamma_{r-1} q+\gamma_{r}\right) & y(k) \\
& =\left(1+\gamma_{1}+\cdots+\gamma_{r-1}+\gamma_{r}\right) y_{s p}(k)
\end{aligned}
$$

that is, Eq. 33. Q.E.D.

Proof of Theorem 2. Define the auxiliary variable:

$$
v(k)=\frac{C_{c} \xi(k)+D_{c}\left[y_{s p}(k)-y(k)\right]}{\beta_{0}}
$$

Then, Eq. 36 can be viewed as being composed of two subsystems:

$\left\{\begin{aligned} \xi(k+1) & =A_{c} \xi(k)+B_{c}\left[y_{s p}(k)-y(k)\right] \\ v(k) & =\frac{C_{c} \xi(k)+D_{c}\left[y_{s p}(k)-y(k)\right]}{\beta_{0}}\end{aligned}\right.$

$$
\left\{\begin{array}{l}
w(k+1)=\Phi\left[w(k), \Psi_{o}\left\{w(k), \beta_{0} v(k)-\sum_{\ell=1}^{r} \beta_{p} h^{r-\ell}[w(k)]\right\}\right] \\
u(k)=\Psi_{o}\left\{w(k), \beta_{0} v(k)-\sum_{\ell=1}^{r} \beta_{p} h^{r-\ell}[w(k)]\right\}
\end{array}\right.
$$

The subsystem of Eq. A5 is the realization of external controller of Eq. 31, whose input/output behavior can be described by the difference operator representation:

$$
\begin{aligned}
& \frac{\left(q^{r}-1\right)+\gamma_{1}\left(q^{r-1}-1\right)+\cdots+\gamma_{r-1}(q-1)}{1+\gamma_{1}+\cdots+\gamma_{r-1}+\gamma_{r}} v(k) \\
& =\frac{1}{\beta_{0}}\left(q^{r}+\beta_{1} q^{r-1}+\cdots+\beta_{r}\right)\left[y_{s p}(k)-y(k)\right]
\end{aligned}
$$

On the other hand, the subsystem of Eq. A6 consists of an input/output linearizing feedback law, with the states being reconstructed via the open-loop observer of Eq. 34. Hence, under consistent initialization of $w$ and $x[w(0)=x(0)]$, we will have $w(k)=x(k)$ for all $k$, and therefore, Eq. A6 induces the dynamics:

$$
y(k+r)+\sum_{\ell=1}^{r} \beta_{\ell} y(k+r-\ell)=\beta_{0} v(k)
$$

or, equivalently,

$$
\frac{1}{\beta_{0}}\left(q^{r}+\beta_{1} q^{r-1}+\cdots+\beta_{r-1} q+\beta_{r}\right) y_{s p}(k)=v(k)
$$

Combining Eqs. A7 and A8, we obtain the desired closed-loop input/output dynamics:

$$
\begin{aligned}
\left(q^{r}+\gamma_{1} q^{r-1}+\cdots+\gamma_{r-1} q+\gamma_{r}\right) & y(k) \\
& =\left(1+\gamma_{1}+\cdots+\gamma_{r-1}+\gamma_{r}\right) y_{s p}(k)
\end{aligned}
$$

that is, Eq. 33. Q.E.D.

Proof of Corollary. At first observe that, from the statespace realization of Eq. 31 of the external controller:

$$
\begin{aligned}
v(k) & =\frac{1}{\beta_{0}}\left[\xi_{1}(k+1)+\sum_{\ell=1}^{r} \beta_{\ell} \xi(k)\right] \\
& =\frac{1}{\beta_{0}}\left[\xi_{r}(k+r)+\sum_{\ell=1}^{r} \beta_{\ell} \xi_{r}(k+r-\ell)\right]
\end{aligned}
$$

On the other hand, from Eqs. 34 and 35, we see that

$$
h[w(k+r)]+\sum_{\ell=1}^{r} \beta_{\ell} h[w(k+r-\ell)]=\beta_{0} v(k)
$$

Combining Eqs. A9 and A10, we conclude that: 


$$
\begin{aligned}
h[w(k+r)]+\sum_{\ell=1}^{r} \beta_{\ell} h[w(k+r-\ell)] & \\
& =\xi_{r}(k+r)+\sum_{\ell=1}^{r} \beta_{\ell} \xi_{r}(k+r-\ell)
\end{aligned}
$$

Consequently, if the external controller and the observer are consistently initialized according to:

$$
\xi(0)=h^{r-\ell}(w(0)), \quad \ell=1, \cdots, r
$$

that is,

$$
\xi_{r}(r-\ell)=h(w(r-\ell)), \quad \ell=1, \cdots, r
$$

we will have:

$$
\xi_{r}(k)=h(w(k)), \quad \forall k,
$$

and therefore,

$$
\xi_{(}(k)=h^{r-\ell}(w(k)), \quad \forall k, \quad \ell=1, \cdots, r
$$

Substituting this into the controller of Eq. 36, we obtain Eq. 37. Q.E.D.

Proof of Theorem 3. For the dead-time free process of Eq. 40, the state feedback of Eq. 22 takes the form:

$$
u(k)=\Psi_{o}\left[\zeta(k), \beta_{0} v(k)-\beta_{1} \zeta_{1}^{(1)}(k)\right]
$$

or

$$
u(k)=\Psi_{o}\left(\left[\begin{array}{c}
\zeta^{(0)} \\
y(k)
\end{array}\right], \beta_{0} v(k)-\beta_{1} y(k)\right)
$$

where the external input $v(k)$ in Eq. A12 is obtained from the external controller (Eq. 31), which for the case $r=1$, simplifies into:

$$
\begin{aligned}
& \xi(k+1)=\xi(k)+\left(1+\gamma_{1}\right) e(k) \\
& v(k)=\frac{\left(1+\beta_{1}\right) \xi(k)+\left(1+\gamma_{1}\right) e(k)}{\beta_{0}}
\end{aligned}
$$

The state feedback of Eq. A12 with $n-1$ states $\xi^{(0)}$ reconstructed from the reduced observer (Eq. 41) induces the closedloop response:

$$
\left(q+\beta_{1}\right) y(k)=\beta_{0} v(k)
$$

On the other hand, the external controller (Eq. A13) can be equivalently written as:

$$
\frac{q-1}{1+\gamma_{1}} v(k)=\frac{1}{\beta_{0}}\left(q+\beta_{1}\right)\left[y_{s p}(k)-y(k)\right]
$$

Combining Eqs. A14 and A15, we obtain the requested closedloop input/output dynamics in Theorem 3. Q.E.D.

Manuscript received Nov. 18, 1991, and revision received June 15, 1992. 